\title{
Piezoelectric Effect in Rochelle Salt
}

\author{
Andriy Andrusyk \\ Institute for Condensed Matter Physics \\ Ukraine
}

\section{Introduction}

For the last several years there has been significant progress in the development of new piezoelectric materials (relaxor ferroelectric single crystals (Park \& Shrout, 1997), solid solutions with high transition temperature (Zhang et al., 2003), lead-free materials (Saito et al., 2004)) and in understanding of mechanisms of the piezoelectric coupling in ferroelectric piezoelectrics (Fu \& Cohen, 2000; Guo et al., 2000). This progress was triggered in particular by the wide use of piezoelectric effect in a variety of devices (resonators, tactile sensors, bandpass filters, ceramic discriminators, SAW filters, piezoresponse force microscopes and others).

What concerns theoretical study of the piezoelectric effect, significant efforts were made in first-principles calculations. Such calculations are possible for the ferroelectrics with a relatively simple structure, in particular for simple and complex perovskites (Bellaiche et al., 2000; Garcia \& Vanderbilt, 1998). For compounds with a complex structure often only the research within the Landau theory is possible. The structure complexity justifies the application of semimicroscopic models considering only that characteristic feature of the microscopic structure which is crucial in explaining the ferroelectric transition or the piezoelectric effect. Such models are adequate for the crystal under study if they are able to explain the wide range of physical properties.

In this chapter sodium potassium tartrate tetrahydrate $\mathrm{NaKC}_{4} \mathrm{H}_{4} \mathrm{O}_{6} \cdot 4 \mathrm{H}_{2} \mathrm{O}$ (Rochelle salt or RS) is studied on the base of the semimicroscopic Mitsui model.

The microscopic mechanism of ferroelectric phase transitions in RS was the subject of numerous investigations. Studies based on x-ray diffraction data (Shiozaki et al., 1998) argued that these were the order-disorder motions of $\mathrm{O} 9$ and $\mathrm{O} 10$ groups, coupled with the displacive vibrations of O8 groups, which were responsible for the phase transitions in Rochelle salt as well as for the spontaneous polarization. Later it was confirmed by the inelastic neutron scattering data (Hlinka et al., 2001). Respective static displacements initiate the emergence of dipole moments in local structure units in ferroelectric phase. Such displacements can be interpreted also as changes in the population ratio of two equilibrium positions of sites in the paraelectric structure (revealed in the structure studies (Noda et al., 2000; Shiozaki et al., 2001)). The order-disorder pattern of phase transitions in RS forms the basis of the semimicroscopic Mitsui model (Mitsui, 1958). In this model the asymmetry of occupancy of double local atomic positions and compensation of electric dipole moments occurring in paraelectric phases were taken into account. 
Recently (Levitskii et al., 2003) Mitsui model as applied to RS was extended by accounting of the piezoelectric coupling between the order parameter and strain $\varepsilon_{4}$. Later this model was extended to the four sublattice type (Levitskii et al., 2009; Stasyuk \& Velychko, 2005) that gives more thorough consideration of real RS structure. We performed our research of piezoelectric effect in Rochelle salt on the basis of the Mitsui-type model containing additional term of transverse field type responsible for dynamic flipping of structural elements (Levitskii, Andrusyk \& Zachek, 2010; Levitskii, Zachek \& Andrusyk, 2010). Originally, this term was added with the aim to describe resonant dielectric response which takes place in RS in submillimeter region. First, we provide characteristics of ferroelectric phase transitions in RS and experimental data for constants of physical properties of RS. Then, we present our study results (thermodynamic and dynamic characteristics) obtained within Mitsui model for RS. Specifically, we calculate permittivity of free and clamped crystals, calculate piezoelectric stress coefficient $e_{14}$, elastic constant $c_{44}^{E}$. The key attention is given to investigation of the phenomenon of piezoelectric resonance.

\section{Physical properties of Rochelle salt}

Rochelle salt (RS), $\mathrm{NaKC}_{4} \mathrm{H}_{4} \mathrm{O}_{6} \cdot 4 \mathrm{H}_{2} \mathrm{O}$ is the oldest and has been for a long time the only known ferroelectrics. RS has been the subject of numerous studies over the past 60 years. It is known for its remarkable ferroelectric state between two Curie points $T_{c 1}=255 \mathrm{~K}$ and $T_{c 2}=297 \mathrm{~K}$ (Jona \& Shirane, 1965). Second order phase transitions occur at both Curie points. The crystalline structure of RS proved to be complex. It is orthorhombic (space group $D_{2}^{3}-P 2_{1} 2_{1} 2$ ) in the paraelectric phases and monoclinic (space group $C_{2}^{2}-P 2_{1}$ ) in the ferroelectric phase (Solans et al., 1997). Spontaneous polarization is directed along the $a$ crystal axis; it is accompanied by a spontaneous shear strain $\varepsilon_{4}$. There are four formula units $(Z=4$; 112 atoms) in the unit cell of Rochelle salt in both ferroelectric and paraelectric phase. In recent study (Görbitz \& Sagstuen, 2008) the complete Rochelle salt structure in paraelectric phase was described.

Due to the symmetry of RS crystal structure some elements of material tensors are zeroes. In RS case material tensors in Voigt index notations are of the form presented below (Shuvalov, 1988).

Elastic stiffnesses or elastic constants $\left(c_{i j}^{E}=\left(\partial \sigma_{i} / \partial \varepsilon_{j}\right)_{E}\right)$ :

$$
\left(c^{E}\right)=\left(\begin{array}{cccccc}
c_{11} & c_{12} & c_{13} & c_{14} & 0 & 0 \\
c_{12} & c_{22} & c_{23} & c_{24} & 0 & 0 \\
c_{13} & c_{23} & c_{33} & c_{34} & 0 & 0 \\
c_{14} & c_{24} & c_{34} & c_{44} & 0 & 0 \\
0 & 0 & 0 & 0 & c_{55} & c_{56} \\
0 & 0 & 0 & 0 & c_{56} & c_{66}
\end{array}\right)
$$

Coefficients of piezoelectric stress $\left(e_{i j}=\left(\partial P_{i} / \partial \varepsilon_{j}\right)_{E}=-\left(\partial \sigma_{j} / \partial E_{i}\right)_{\varepsilon}\right)$ :

$$
(e)=\left(\begin{array}{cccccc}
e_{11} & e_{12} & e_{13} & e_{14} & 0 & 0 \\
0 & 0 & 0 & 0 & e_{25} & e_{26} \\
0 & 0 & 0 & 0 & e_{35} & e_{36}
\end{array}\right)
$$


Dielectric permittivity $\left(\chi_{i j}^{\alpha}=\left(\partial P_{i} / \partial E_{j}\right)_{\alpha}\right.$, where $\alpha$ is $\sigma$ or $\left.\varepsilon\right)$ :

$$
\left(\chi^{\sigma, \varepsilon}\right)=\left(\begin{array}{ccc}
\chi_{11} & 0 & 0 \\
0 & \chi_{22} & \chi_{23} \\
0 & \chi_{23} & \chi_{33}
\end{array}\right)
$$

It is necessary to make some comments about the notations. Superscripts to the matrices of physical properties indicate that a physical characteristic denoted by a superscript is constant or zero (for instance $(c)$ with superscript $E$ denotes matrix of elastic constants at constant electric field: $\left.\left(c^{E}\right)\right)$. We omitted superscripts $E$ and $\sigma, \varepsilon$ for components of tensors $\left(c^{E}\right)$ and $\left(\chi^{\sigma, \varepsilon}\right)$ respectively but keep them in mind. Notation $\left(\chi^{\sigma, \varepsilon}\right)$ denotes two different tensors: $\left(\chi^{\sigma}\right)$ and $\left(\chi^{\varepsilon}\right)$ which we will call tensors of dielectric permittivity at constant stress and strain respectively. We will also call them tensor of free crystal dielectric permittivity (zero stress is assumed) and of clamped crystal dielectric permittivity. Hereinafter coefficients equal to zero in paraelectric phases are presented in bold.

Experimental data for physical constants are presented in Figs. 1, 2, and 3.
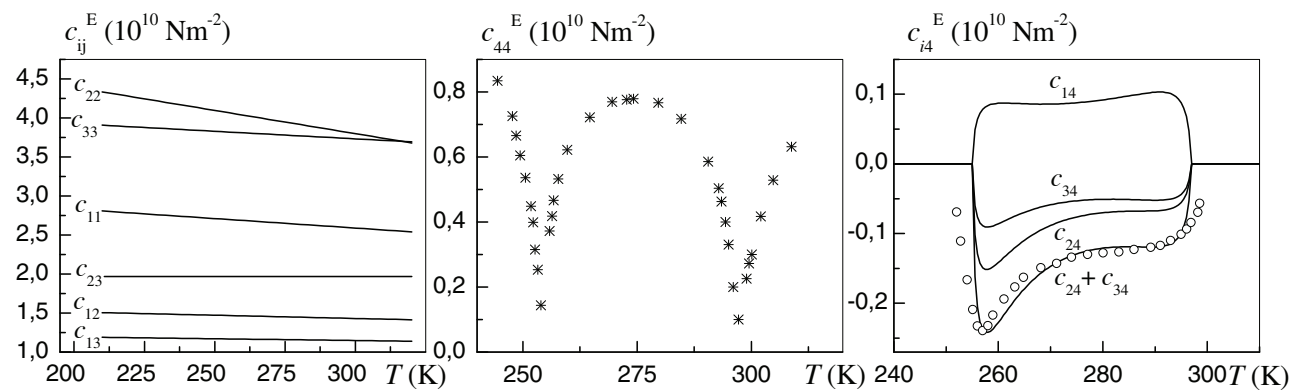

Fig. 1. Rochelle salt elastic constants. Solid lines for $c_{i j}(i, j=1,2,3)$ are experimental data (Mason, 1950), * correspond to $c_{44}$ (Yu. Serdobolskaya, 1996), o correspond to $c_{24}^{E}+c_{34}^{E}$ (Shiozaki et al., 2006). Lines for $c_{14}^{E}, c_{24}^{E}, c_{34}^{E}$ are the results of theoretical calculations (Levitskii et al., 2005).
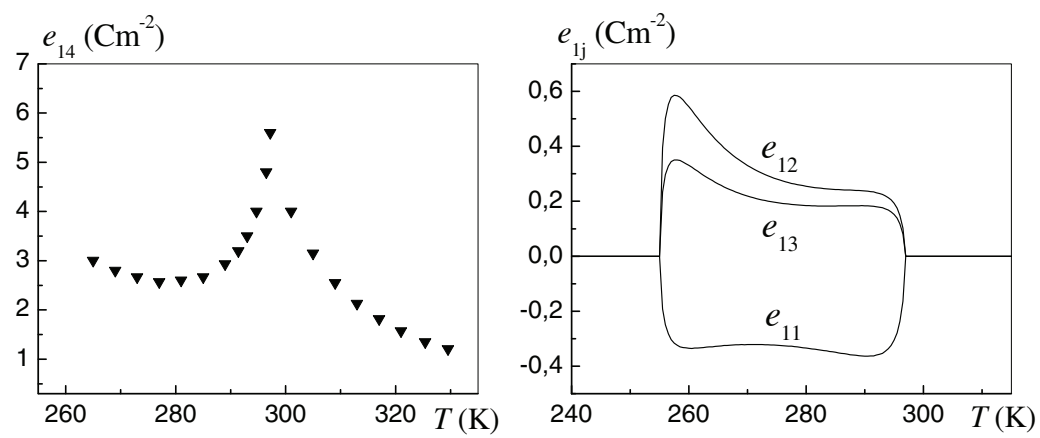

Fig. 2. Rochelle salt coefficients of piezoelectric stress. Points $\boldsymbol{\nabla}$ are experimental data (Beige $\&$ Kühnel, 1984) for $e_{14}$. Solid lines correspond to the results of theoretical calculations $e_{1 j}$ $(j=1,2,3)$ (Levitskii et al., 2005). 

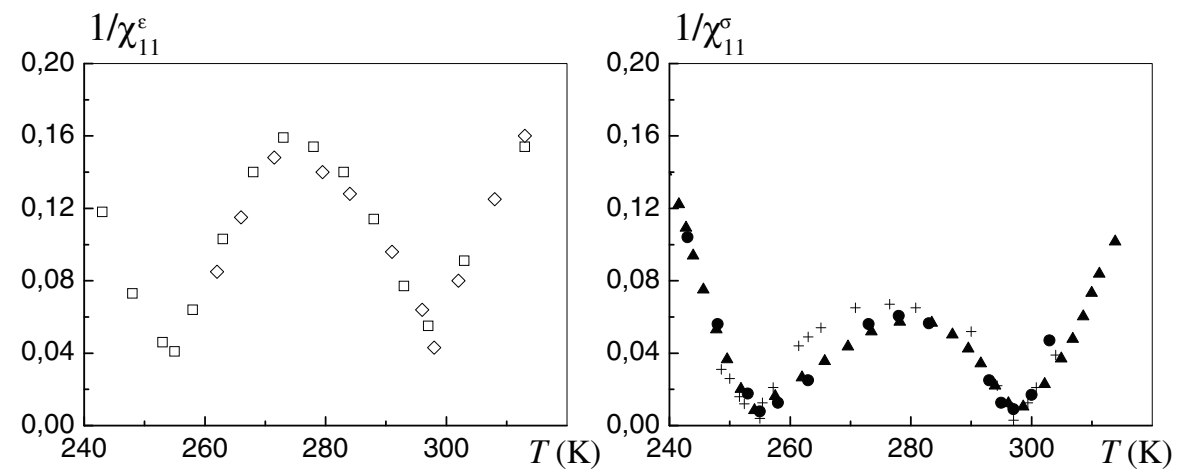

Fig. 3. Experimental data for clamped and free crystal inverse dielectric permittivity of Rochelle salt. $1 / \chi_{11}^{\varepsilon}: \square$ (Sandy \& Jones, 1968), $\diamond$ (Mueller, 1935). 1/ $\chi_{11}^{\sigma}:+($ Yurin, 1965), • (Mason, 1939), \ (Taylor et al., 1984).

As Fig. 1 shows elastic constants $c_{i j}^{E}(i, j=1,2,3)$ do not change their behaviour in phase transition points, whereas constant $c_{44}^{E}$ becomes zero at the transition point. Besides, $c_{44}$ is strongly dependent on $T$ as $c_{i j}^{E}(i, j=1,2,3)$ are almost independent of $T$. Constants $c_{14}^{E}, c_{24}^{E}$, $c_{34}^{E}$, as it is expected on symmetry grounds, are equal to zero in paraelectric phases. There are no experiments for $c_{14}^{E}$ and there are no experiments for $c_{24}^{E}, c_{34}^{E}$ measured separately. There is only the experiment for $c_{24}^{E}+c_{34}^{E}$ (Shiozaki et al., 2006). However, $c_{14}^{E}, c_{24}^{E}$, and $c_{34}^{E}$ were estimated theoretically (Levitskii et al., 2005) and the result of estimation is presented in Fig. 1. Correspondent result for $c_{24}^{E}+c_{34}^{E}$ is compared to experimental data where good agreement was derived.

Fig. 2 presents temperature dependencies of piezoelectric stress coefficients. As one can see coefficients $e_{11}, e_{12}, e_{13}$ differ from zero only inside ferroelectric phase. Coefficient $e_{14}$ has sharp (but finite) peak in the transition point, whereas coefficients $e_{11}, e_{12}, e_{13}$ do not have it. Free and clamped crystal longitudinal susceptibilities are presented in Fig. 3. Free crystal susceptibility has singularities in transition point, whereas clamped susceptibility remains finite.

\section{Thermodynamic characteristics of Rochelle salt}

\subsection{Theoretical study of the Mitsui model}

We give consideration to a two-sublattice order-disorder type system with an asymmetric double-well potential. Hamiltonian of such system is referred to as the Mitsui Hamiltonian. We assume this system has essential piezoelectric coupling of the order parameter with component of strain tensor $\varepsilon_{4}$ which should be accounted. We suppose the polarization is directed along $x$-axes and arises due to the structural units ordering in the one of two possible equilibrium positions. Precisely this case occurs in RS and such modified Mitsui model was considered earlier (Levitskii et al., 2003). We complement this model with transverse field to take into account the possibility of dynamic ordering units flipping between two equilibrium 
positions. The resulting Hamiltonian is of the following form:

$$
H=U_{0}-\sum_{q, q^{\prime}}\left[\frac{J_{q q^{\prime}}}{2}\left(S_{q 1}^{z} S_{q^{\prime} 1}^{z}+S_{q 2}^{z} S_{q^{\prime} 2}^{z}\right)+K_{q q^{\prime}} S_{q 1}^{z} S_{q^{\prime} 2}^{z}\right]-\sum_{q f}\left[\Omega S_{q f}^{x}+\left(\Delta_{f}-2 \psi_{14} \varepsilon_{4}+\mu E_{1}\right) S_{q f}^{z}\right] .
$$

where

$$
U_{0}=N\left[\frac{1}{2} v c_{44}^{E 0} \varepsilon_{4}^{2}-v e_{14}^{0} E_{1} \varepsilon_{4}-\frac{1}{2} v \chi_{11}^{\varepsilon 0} E_{1}^{2}\right] .
$$

represent the elastic, piezoelectric, and electric energies attributed to a host lattice, in which potential the pseudospin moves (with the 'seed' elastic constant $c_{44}^{E 0}$, the coefficient of piezoelectric stress $e_{14}^{0}$, and dielectric susceptibility $\left.\chi_{11}^{\varepsilon 0}\right) ; v$ is a volume of cell, containing a pair of pseudospins (ordering units or dipoles) of one lattice site $\mathbf{q}$ and different sublattices $f=1,2$ (further we will call it a unit cell ${ }^{1}$ ), and $N$ is a number of unit cells. The first sum describes direct interaction of the ordering units: $J_{q q^{\prime}}=J_{q^{\prime} q}$ and $K_{q q^{\prime}}=K_{q^{\prime} q}$ are interaction potentials between pseudospins belonging to the same and to different sublattices, respectively. The first term in the second sum is the transverse field; the second term describes a) energy, associated with asymmetry of the potential, where $\Delta_{f}$ is asymmetry parameter: $\Delta_{1}=-\Delta_{2}=\Delta, \mathrm{b}$ ) interaction energy of pseudospin with the field, arising due to the piezoelectric deformation $\varepsilon_{4}$ and $\psi_{14}$ is parameter of piezoelectric coupling, c) interaction energy of pseudospin with external electric field $E_{1}$, where $\mu$ is effective dipole moment of the model unit cell.

We conduct the study within the mean field approximation (MFA). Performing identical transformation

$$
S_{q f}^{z}=\left\langle S_{q f}^{z}\right\rangle+\Delta S_{q f}^{z}
$$

and neglecting the quadratic fluctuations, we rewrite the initial Hamiltonian (4) as

$$
H_{\mathrm{MFA}}=U_{0}+\sum_{q q^{\prime}}\left[\frac{J_{q q^{\prime}}}{2}\left(\left\langle S_{q 1}^{z}\right\rangle\left\langle S_{q^{\prime} 1}^{z}\right\rangle+\left\langle S_{q 2}^{z}\right\rangle\left\langle S_{q^{\prime} 2}^{z}\right\rangle\right)+K_{q q^{\prime}}\left\langle S_{q 1}^{z}\right\rangle\left\langle S_{q^{\prime 2}}^{z}\right\rangle\right]-\sum_{q f} \mathcal{H}_{q f} \boldsymbol{S}_{q f},
$$

where $\mathcal{H}_{q f}$ are the mean local fields having effect on the pseudospins $\boldsymbol{S}_{q f}$ :

$$
\begin{gathered}
\mathcal{H}_{q f}^{x}=\Omega, \quad \mathcal{H}_{q f}^{y}=0, \quad \mathcal{H}_{q f}^{z}=h_{q f}, \\
h_{q 1}=\sum_{q^{\prime}}\left[J_{q q^{\prime}}\left\langle S_{q^{\prime} 1}^{z}\right\rangle+K_{q q^{\prime}}\left\langle S_{q^{\prime} 2}^{z}\right\rangle\right]+\Delta-2 \psi_{14} \varepsilon_{4}+\mu E_{1} \\
h_{q 2}=\sum_{q^{\prime}}\left[J_{q q^{\prime}}\left\langle S_{q^{\prime} 2}^{z}\right\rangle+K_{q q^{\prime}}\left\langle S_{q^{\prime} 1}^{z}\right\rangle\right]-\Delta-2 \psi_{14} \varepsilon_{4}+\mu E_{1} .
\end{gathered}
$$

Within MFA we can calculate mean equilibrium values of the pseudospin operators:

$$
\left\langle\boldsymbol{S}_{q f}\right\rangle=\operatorname{Sp}\left(\boldsymbol{S}_{q f} \rho_{\mathrm{MFA}}\right),
$$

\footnotetext{
${ }^{1}$ Actual unit cell of the Rochelle salt crystal contains two pairs of pseudospins of two lattice sites and different sublattices; therefore, we should set the value of the model unit cell volume to be half of the crystal unit cell volume.
} 
where

$$
\rho_{\mathrm{MFA}}=\frac{\exp \left(-\frac{H_{\mathrm{MFA}}}{k_{B} T}\right)}{\operatorname{Sp} \exp \left(-\frac{H_{\mathrm{MFA}}}{k_{B} T}\right)}
$$

and $k_{B}$ is the Boltzmann constant. After calculations we derive

$$
\left\langle\boldsymbol{S}_{q f}\right\rangle=\frac{1}{2} \frac{\mathcal{H}_{q f}}{\mathcal{H}_{q f}} \tanh \frac{\mathcal{H}_{q f}}{2 k_{B} T},
$$

where $\mathcal{H}_{q f} \equiv\left|\mathcal{H}_{q f}\right|=\lambda_{q f}=\sqrt{\Omega^{2}+h_{q f}^{2}}$.

Free energy of a crystal within MFA

$$
F(4)=-k_{B} T \ln \operatorname{Sp} \exp \left(-\frac{H_{\mathrm{MFA}}}{k_{B} T}\right)
$$

is following:

$$
\begin{aligned}
F(4)= & U_{0}+\sum_{q q^{\prime}}\left[\frac{J_{q q^{\prime}}}{2}\left(\left\langle S_{q 1}^{z}\right\rangle\left\langle S_{q^{\prime} 1}^{z}\right\rangle+\left\langle S_{q 2}^{z}\right\rangle\left\langle S_{q^{\prime 2}}^{z}\right\rangle\right)+K_{q q^{\prime}}\left\langle S_{q 1}^{z}\right\rangle\left\langle S_{q^{\prime 2}}^{z}\right\rangle\right] \\
& -k_{B} T \sum_{q f} \ln \left(2 \cosh \frac{\lambda_{q f}}{2 k_{B} T}\right) .
\end{aligned}
$$

In homogeneous external field the system of $6 \mathrm{~N}$ equations (11) has a lot of solutions, with a homogeneous one among others: $\left\langle\boldsymbol{S}_{q f}\right\rangle \equiv\left\langle\boldsymbol{S}_{q f}\right\rangle_{0} \neq f(q)$. In case of a Rochelle salt crystal we have $J_{q q^{\prime}}>0, K_{q q^{\prime}}>0$ and it is homogeneous solution which provides free energy minimum. In this case, system of equations reduces into system

$$
\left\langle\boldsymbol{S}_{q f}\right\rangle_{0}=\frac{1}{2} \frac{\mathcal{H}_{f}^{(0)}}{\mathcal{H}_{f}^{(0)}} \tanh \frac{\mathcal{H}_{f}^{(0)}}{2 k_{B} T^{\prime}}
$$

where the local field $\mathcal{H}_{f}^{(0)}$ is following:

$$
\begin{gathered}
\mathcal{H}_{f}^{(0) x}=\Omega, \quad \mathcal{H}_{f}^{(0) y}=0, \quad \mathcal{H}_{f}^{(0) z}=h_{f}, \\
h_{1}=J_{0}\left\langle S_{q 1}^{z}\right\rangle_{0}+K_{0}\left\langle S_{q 2}^{z}\right\rangle_{0}+\Delta-2 \psi_{14} \varepsilon_{4}+\mu E_{1}, \\
h_{2}=J_{0}\left\langle S_{q 2}^{z}\right\rangle_{0}+K_{0}\left\langle S_{q 1}^{z}\right\rangle_{0}-\Delta-2 \psi_{14} \varepsilon_{4}+\mu E_{1},
\end{gathered}
$$

where

$$
J_{0}=\sum_{q^{\prime}} J_{q q^{\prime}}, \quad K_{0}=\sum_{q^{\prime}} K_{q q^{\prime}} ; \quad \mathcal{H}_{f}^{(0)} \equiv\left|\mathcal{H}_{f}^{(0)}\right|=\lambda_{f}, \quad \lambda_{f}=\sqrt{\Omega^{2}+h_{f}^{2}} .
$$

$z$-component of this equation system forms two-equation system $(f=1,2)$ to determine $\left\langle S_{q f}^{z}\right\rangle_{0}$ :

$$
\left\langle S_{q f}^{z}\right\rangle_{0}=\frac{h_{f}}{2 \lambda_{f}} \tanh \frac{\lambda_{f}}{2 k_{B} T} .
$$


Having introduced new variables

$$
\boldsymbol{\xi}=\left\langle\boldsymbol{S}_{q 1}\right\rangle_{0}+\left\langle\boldsymbol{S}_{q 2}\right\rangle_{0}, \quad \boldsymbol{\sigma}=\left\langle\boldsymbol{S}_{q 1}\right\rangle_{0}-\left\langle\boldsymbol{S}_{q 2}\right\rangle_{0}
$$

( $\xi^{z}$ and $\sigma^{z}$ are ferroelectric and antiferroelectric ordering parameters), we obtain system of equations (15) in a form:

$$
\left\{\begin{array}{l}
\xi^{z}=\frac{1}{2}\left[\frac{\tilde{h}_{1}}{\tilde{\lambda}_{1}} \tanh \frac{\tilde{\lambda}_{1}}{2 T}+\frac{\tilde{h}_{2}}{\tilde{\lambda}_{2}} \tanh \frac{\tilde{\lambda}_{2}}{2 T}\right], \\
\sigma^{z}=\frac{1}{2}\left[\frac{\tilde{h}_{1}}{\tilde{\lambda}_{1}} \tanh \frac{\tilde{\lambda}_{1}}{2 T}-\frac{\tilde{h}_{2}}{\tilde{\lambda}_{2}} \tanh \frac{\tilde{\lambda}_{2}}{2 T}\right],
\end{array}\right.
$$

where unknowns $\xi^{z}$ and $\sigma^{z}$ are defined at given $T, E_{1}, \varepsilon_{4}$. In system (17) the following notations are used:

$$
\begin{aligned}
& \tilde{h}_{f}=h_{f} / k_{B}, \quad \tilde{\lambda}_{f}=\sqrt{\tilde{\Omega}^{2}+\tilde{h}_{f}^{2}}, \\
& \tilde{h}_{1}=\tilde{R}_{0}^{+} \xi^{z}+\tilde{R}_{0}^{-} \sigma^{z}+\tilde{\Delta}-2 \tilde{\psi}_{14} \varepsilon_{4}+\tilde{\mu} E_{1}, \\
& \tilde{h}_{2}=\tilde{R}_{0}^{+} \xi^{z}-\tilde{R}_{0}^{-} \sigma^{z}-\tilde{\Delta}-2 \tilde{\psi}_{14} \varepsilon_{4}+\tilde{\mu} E_{1} .
\end{aligned}
$$

Here

$$
\tilde{\Omega}=\frac{\Omega}{k_{B}}, \tilde{\Delta}=\frac{\Delta}{k_{B}}, \quad \tilde{\psi}_{14}=\frac{\psi_{14}}{k_{B}}, \tilde{\mu}=\frac{\mu}{k_{B}}, \tilde{R}_{0}^{ \pm}=\frac{\tilde{J}_{0} \pm \tilde{K}_{0}}{2}, \tilde{J}_{0}=\frac{J_{0}}{k_{B}}, \quad \tilde{K}_{0}=\frac{K_{0}}{k_{B}} .
$$

The system (17) is system of two equations with unknowns $\xi^{z}$ and $\sigma^{z}$.

When values $\xi^{z}$ and $\sigma^{z}$ are defined, we can calculate values $\xi^{x}$ and $\sigma^{x}$ using (13) and (16):

$$
\xi^{x}=\frac{1}{2}\left[\frac{\tilde{\Omega}}{\tilde{\lambda}_{1}} \tanh \frac{\tilde{\lambda}_{1}}{2 T}+\frac{\tilde{\Omega}}{\tilde{\lambda}_{2}} \tanh \frac{\tilde{\lambda}_{2}}{2 T}\right], \quad \sigma^{x}=\frac{1}{2}\left[\frac{\tilde{\Omega}}{\tilde{\lambda}_{1}} \tanh \frac{\tilde{\lambda}_{1}}{2 T}-\frac{\tilde{\Omega}}{\tilde{\lambda}_{2}} \tanh \frac{\tilde{\lambda}_{2}}{2 T}\right] ;
$$

values $\xi^{y}, \sigma^{y}$ are equal to zero.

Free energy per model unit cell $f(4)=F(4) /\left(k_{B} N\right)$ in variables $\xi^{z}$ and $\sigma^{z}$ is following:

$$
f(4)=\frac{\tilde{v}}{2} c_{44}^{E 0} \varepsilon_{4}^{2}-\tilde{v} e_{14}^{0} \varepsilon_{4} E_{1}-\frac{\tilde{v}}{2} \chi_{11}^{\varepsilon 0} E_{1}^{2}+\frac{\tilde{R}_{0}^{+}}{2}\left(\xi^{z}\right)^{2}+\frac{\tilde{R}_{0}^{-}}{2}\left(\sigma^{z}\right)^{2}-T \sum_{f} \ln \left(2 \cosh \frac{\tilde{\lambda}_{f}}{2 T}\right),
$$

where $\tilde{v}=\frac{v}{k_{B}}$. By differentiating free energy we can find dielectric, elastic, and piezoelectric properties of the Rochelle salt.

The conditions

$$
\frac{1}{\tilde{v}}\left(\frac{\partial f(4)}{\partial \varepsilon_{4}}\right)_{E_{1}, T}=\sigma_{4}, \quad \frac{1}{\tilde{v}}\left(\frac{\partial f(4)}{\partial E_{1}}\right)_{\varepsilon_{4}, T}=-P_{1}
$$

yield the following expression for stress $\sigma_{4}$ and polarization $P_{1}$ :

$$
\begin{aligned}
& \sigma_{4}=c_{44}^{E 0} \varepsilon_{4}-e_{14}^{0} E_{1}+\frac{2 \tilde{\psi}_{14}}{\tilde{v}} \xi^{z}, \\
& P_{1}=e_{14}^{0} \varepsilon_{4}+\chi_{11}^{\varepsilon 0} E_{1}+\frac{\tilde{\mu}}{\tilde{v}} \xi^{z} .
\end{aligned}
$$


Independent variable is stress rather than deformation, so we need express local fields in terms of $\sigma_{4}$ when solving system (17). Having used (21a) we derive

$$
\varepsilon_{4}=\frac{\sigma_{4}}{c_{44}^{E 0}}+\frac{e_{14}^{0}}{c_{44}^{E 0}} E_{1}-\frac{2 \tilde{\psi}_{14}}{\tilde{v} c_{44}^{E 0}} \xi^{z} .
$$

On the basis of Eq. (22) we can rewrite local fields in the following way:

$$
\begin{aligned}
& \tilde{h}_{1}=\tilde{R}_{0}^{\prime+} \xi^{z}+\tilde{R}_{0}^{-} \sigma^{z}+\tilde{\Delta}-\frac{2 \tilde{\psi}_{14}}{c_{44}^{E 0}} \sigma_{4}+\tilde{\mu}^{\prime} E_{1}, \\
& \tilde{h}_{2}=\tilde{R}_{0}^{\prime+} \xi^{z}-\tilde{R}_{0}^{-} \sigma^{z}-\tilde{\Delta}-\frac{2 \tilde{\psi}_{14}}{c_{44}^{E 0}} \sigma_{4}+\tilde{\mu}^{\prime} E_{1},
\end{aligned}
$$

where $\tilde{R}^{\prime+}$ and $\tilde{\mu}^{\prime}$ are following:

$$
\tilde{R}_{0}^{\prime+}=\tilde{R}_{0}^{+}+\frac{4 \tilde{\psi}_{14}^{2}}{\tilde{v} c_{44}^{E 0}}, \quad \tilde{\mu}^{\prime}=\tilde{\mu}-\frac{2 \tilde{\psi}_{14} e_{14}^{0}}{c_{44}^{E 0}} .
$$

System (17) with local fields (23), considered at $\sigma_{4}=0, E_{1}=0$, has solutions of two types: $\xi^{z}=0$ and $\xi^{z} \neq 0$. The minimum Helmholtz free energy $\left(g(4)=f(4)-\tilde{v} \sigma_{4} \varepsilon_{4}\right)$ condition defines which of the solutions is actually realized at each particular $T$. The solution of first type describes paraelectric phase and the solution of second type describes ferroelectric phase. In paraelectric phase we have also $\sigma^{x}=0$.

We will calculate elastic, piezoelectric, and dielectric constants by differentiation $P_{1}(21 \mathrm{~b})$ and $\sigma_{4}(21 \mathrm{a})$ at constant $T$ :

$$
\begin{aligned}
& \mathrm{d} P_{1}=e_{14} \mathrm{~d} \varepsilon_{4}+\chi_{11}^{\varepsilon} \mathrm{d} E_{1}, \\
& \mathrm{~d} \sigma_{4}=c_{44}^{E} \mathrm{~d} \varepsilon_{4}-e_{14} \mathrm{~d} E_{1} .
\end{aligned}
$$

Here $\chi_{11}^{\varepsilon}$ is longitudinal dielectric susceptibility at constant strain, $c_{44}^{E}$ is elastic constant at constant field, $e_{14}$ is coefficient of the piezoelectric stress.

The result is following: ${ }^{2}$

$$
\chi_{11}^{\varepsilon}=\left(\frac{\partial P_{1}}{\partial E_{1}}\right)_{\varepsilon_{4}}=\chi_{11}^{\varepsilon 0}+\frac{\tilde{\mu}}{\tilde{v}}\left(\frac{\partial \xi^{z}}{\partial E_{1}}\right)_{\varepsilon_{4}}=\chi_{11}^{\varepsilon 0}+\frac{\tilde{\mu}^{2}}{\tilde{v}} f_{1}\left(T, \sigma_{4}, E_{1}\right),
$$

where

$$
f_{1}\left(T, \sigma_{4}, E_{1}\right)=\frac{e_{1}-\tilde{R}_{0}^{-}\left(e_{1}^{2}-e_{2}^{2}\right)}{1-e_{1}\left(\tilde{R}_{0}^{+}+\tilde{R}_{0}^{-}\right)+\tilde{R}_{0}^{+} \tilde{R}_{0}^{-}\left(e_{1}^{2}-e_{2}^{2}\right)}
$$

and following notations are used:

$$
\begin{aligned}
& e_{1}=\frac{a_{1}+a_{2}}{4 T}+\frac{\tilde{\Omega}^{2}\left(b_{1}+b_{2}\right)}{2}, \quad e_{2}=\frac{a_{1}-a_{2}}{4 T}+\frac{\tilde{\Omega}^{2}\left(b_{1}-b_{2}\right)}{2}, \\
& a_{i}=\frac{\tilde{h}_{i}^{2}}{\tilde{\lambda}_{i}^{2}}-\eta_{i}^{2}, \quad b_{i}=\frac{\eta_{i}}{\tilde{h}_{i} \tilde{\lambda}_{i}^{2}}, \quad(i=1,2) ; \quad \eta_{1}=\xi^{z}+\sigma^{z}, \quad \eta_{2}=\xi^{z}-\sigma^{z} .
\end{aligned}
$$

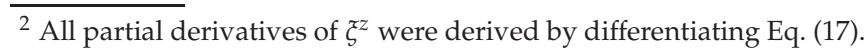


Coefficient of the piezoelectric stress:

$$
e_{14}=\left(\frac{\partial P_{1}}{\partial \varepsilon_{4}}\right)_{E_{1}}=e_{14}^{0}-\frac{2 \tilde{\mu} \tilde{\psi}_{14}}{\tilde{v}} f_{1}\left(T, \sigma_{4}, E_{1}\right)
$$

Elastic constant at constant field:

$$
c_{44}^{E}=\left(\frac{\partial \sigma_{4}}{\partial \varepsilon_{4}}\right)_{E_{1}}=c_{44}^{E 0}-\frac{4 \tilde{\psi}_{14}^{2}}{\tilde{v}} f_{1}\left(T, \sigma_{4}, E_{1}\right) .
$$

Coefficient of the piezoelectric strain $d_{14}=\left(\partial P_{1} / \partial \sigma_{4}\right)_{E_{1}}$ and dielectric susceptibility of free crystal $\chi_{11}^{\sigma}=\left(\partial P_{1} / \partial E_{1}\right)_{\sigma_{4}}$ could be derived through $e_{14}, c_{44}^{E}$ and $\chi_{11}^{\varepsilon}$ :

$$
d_{14}=\frac{e_{14}}{c_{44}^{E}}, \quad \chi_{11}^{\sigma}=\chi_{11}^{\varepsilon}+e_{14} d_{14}
$$

We may notice that at $\tilde{\Omega}=0$ all results presented here coincide with the results of previous calculations (Levitskii et al., 2003), where transverse field was not taken into account.

\subsection{Results of calculations for Rochelle salt}

The proposed model was used for analysis of physical properties of Rochelle salt crystal that is not externally affected $\left(E_{1}=0, \sigma_{4}=0\right)$. To obtain specific numerical results it is necessary first of all to derive theory model parameters for calculations. Deriving procedure was described in (Levitskii, Zachek \& Andrusyk, 2010) and here we will restrict ourselves to parameters presenting:

$$
\begin{aligned}
& \tilde{\Omega}=113.467 \mathrm{~K}, \quad \tilde{J}_{0}=813.216 \mathrm{~K}, \quad \tilde{K}_{0}=1447.17 \mathrm{~K}, \quad \tilde{\Delta}=719.937 \mathrm{~K}, \quad \tilde{\psi}_{4}=-720.0 \mathrm{~K}, \\
& c_{44}^{E 0}=1.224 \times 10^{10} \mathrm{Nm}^{-2}, \quad e_{14}^{0}=31.64 \times 10^{-2} \mathrm{Cm}^{-2}, \quad \chi_{11}^{\varepsilon 0}=0.0, \\
& \mu(T)=a+k(T-297), \quad a=8.157 \times 10^{-30} \mathrm{Cm}, \quad k=-0.0185 \times 10^{-30} \mathrm{CmK}^{-1} .
\end{aligned}
$$

Unit cell volume (volume per two pseudospins from the same site and different sublattices) is $v=5.219 \times 10^{-22} \mathrm{~cm}^{3}$ (Bronowska, 1981).

The results of calculations made for static dielectric characteristics are shown together with experimental data in Fig. 4. The derived agreement is very good considering that Mitsui model is rather inaccurate model of RS and the used MFA is a weak approximation.

Besides, we derived that dielectric permittivity of the free crystal has singularity in the transition points while dielectric permittivity of the clamped crystal doesn't. Elastic constant $c_{44}$ becomes equal to zero at the transition points, coefficient of the piezoelectric stress $e_{14}$ doesn't have singularity in the transition point. All these results agree with the prediction of the Landau theory for the behaviour of physical characteristics in the vicinity of the transition points. However, presented semimicroscopic approach has an advantage over the Landau theory: it allowed to explain physical properties of Rochelle salt in wide temperature rage containing both transition points in natural way. Besides that Mitsui model gives some insight into microscopical mechanism of the phase transition of Rochelle salt. 

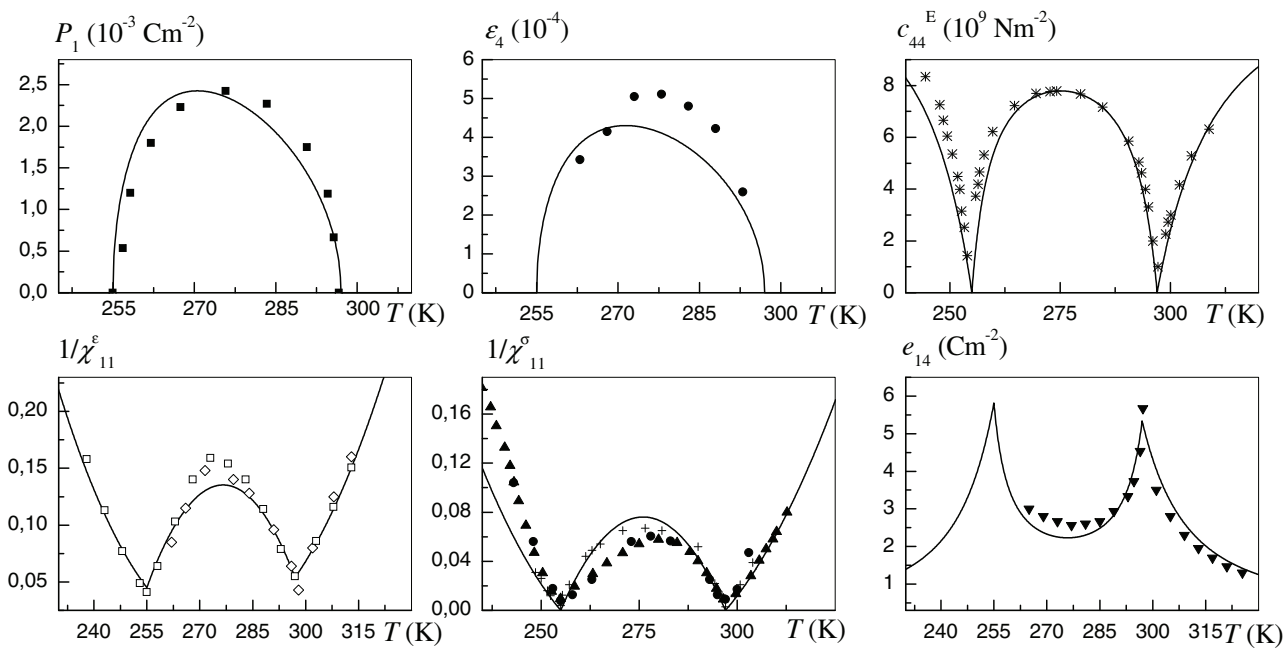

Fig. 4. Theoretical and experimental physical characteristics of Rochelle salt. Solid line corresponds to calculations, Points are the experimental data. $P_{1}(T): \mathbf{a}(\mathrm{Cady}, 1964), \varepsilon_{4}(T)$ : (Ubbelohde \& Woodward, 1946), $c_{44}^{E}: *$ (Yu. Serdobolskaya, 1996), $1 / \chi_{11}^{\varepsilon}(T): \square($ Sandy \& Jones, 1968), $\diamond$ (Mueller, 1935), $1 / \chi_{11}^{\sigma}(T): \Delta$ (Taylor et al., 1984), $e_{14}(T): \boldsymbol{\nabla}$ (Beige \& Kühnel, 1984).

\section{Dynamic properties of Rochelle salt}

\subsection{Order parameter dynamics. Dielectric susceptibility of a clamped crystal}

We consider dynamic properties of the system with Hamiltonian (4) within the Bloch equations method

$$
\hbar \frac{d\left\langle\boldsymbol{S}_{q f}\right\rangle_{t}}{d t}=\left\langle\boldsymbol{S}_{q f}\right\rangle_{t} \times \mathcal{H}_{q f}(t)-\frac{\hbar}{T_{1}}\left[\left\langle\boldsymbol{S}_{q f}\right\rangle_{t \|}-\overline{\left\langle\boldsymbol{S}_{q f}\right\rangle_{t}}\right]
$$

Right part of this equation consists of two terms.

The first term is Heisenberg part of the motion equation, calculated within random phase approximation (RPA), where ' $\times$ ' denotes the vector product and $\mathcal{H}_{q f}(t)$ are the instantaneous values of the local fields: ${ }^{3}$

$$
\mathcal{H}_{q f}^{x}(t)=\Omega, \quad \mathcal{H}_{q f}^{y}=0, \quad \mathcal{H}_{q f}^{z}(t)=h_{q f}(t),
$$

\footnotetext{
${ }^{3}$ Original Heisenberg part of the motion equation is $-\mathrm{i}\left\langle\left[\boldsymbol{S}_{q f}, H\right]\right\rangle_{t}$. Within RPA mean value of commutator with time dependent statistical operator in form of (10) is to be calculated. Doing necessary calculations one can derive that

$$
-\mathrm{i}\left\langle\left[\boldsymbol{S}_{q f}, H\right]\right\rangle_{t}=\left\langle\boldsymbol{S}_{q f}\right\rangle_{t} \times \mathcal{H}_{q f}(t)
$$
}




$$
\begin{aligned}
& h_{q 1}(t)=\sum_{q^{\prime}}\left[J_{q q^{\prime}}\left\langle S_{q^{\prime} 1}^{z}\right\rangle_{t}+K_{q q^{\prime}}\left\langle S_{q^{\prime} 2}^{z}\right\rangle_{t}\right]+\Delta-2 \psi_{14} \varepsilon_{4 q}(t)+\mu E_{1 q}(t), \\
& h_{q 2}(t)=\sum_{q^{\prime}}\left[J_{q q^{\prime}}\left\langle S_{q^{\prime} 2}^{z}\right\rangle_{t}+K_{q q^{\prime}}\left\langle S_{q^{\prime} 1}^{z}\right\rangle_{t}\right]-\Delta-2 \psi_{14} \varepsilon_{4 q}(t)+\mu E_{1 q}(t) .
\end{aligned}
$$

The second term describes relaxation of the pseudospin component $\left\langle\boldsymbol{S}_{q f}\right\rangle_{t \|}$ (longitudinal to the instantaneous value of the local field) towards its quasiequilibrium value with a characteristic time $T_{1} \cdot{ }^{4}$ Quasiequilibrium mean values $\overline{\left\langle\boldsymbol{S}_{q f}\right\rangle}$ te defined as (see Eq. (11)):

$$
{\overline{\left\langle\boldsymbol{S}_{q f}\right\rangle_{t}}}_{t}=\frac{1}{2} \frac{\mathcal{H}_{q f}(t)}{\mathcal{H}_{q f}(t)} \tanh \left[\frac{1}{2 k_{B} T} \mathcal{H}_{q f}(t)\right] .
$$

Relaxation term describes non-equilibrium processes in a pseudospin system. In real situation, a pseudospin system is not an isolated system, whereas it is a part of a larger system. That part of extended system which is not a pseudospin subsystem appears as thermostat that behaves without criticality. Respectively, pseudospin excitations relax due to the interaction with thermostat to their quasiequilibrium values for a characteristic relaxation time $T_{1}$. As far as a phase transition is a collective effect and the relaxation term in Eq. (32) describes individual relaxation of each pseudospin, it becomes clear that relaxation time $T_{1}$ should have no singularity at the Curie point. Relaxation time can be derived $a b$ initio but we consider it to be a model parameter and take it to be independent from temperature.

In the same way it can be explained why relaxation in Eq. (32) occurs towards quasiequilibrium state and not to thermodynamic equilibrium state. Relaxation term describes individual relaxation of pseudospin, which 'is not aware' of the state of thermodynamic equilibrium but 'is aware' of the state of its environment at a particular moment. At every moment this environment creates instantaneous molecular fields which define quasiequilibrium state. Instantaneous quasiequilibrium average of pseudospin operators are defined from Eq. (9), (10) but with molecular fields Eq. (33), (34). Making necessary calculations we obtain quasiequilibrium average $\overline{\left\langle\boldsymbol{S}_{q f}\right\rangle_{t}}$ in form of Eq. (35). Eventually, of course, quasiequilibrium values follow to equilibrium ones and relaxation leads excited system to thermodynamic equilibrium state.

As we are interested in linear response of the system to a small external variable electric field

$$
\delta E_{1 q}(t) \quad\left(E_{1 q}(t)=E_{1}+\delta E_{1 q}(t)\right),
$$

it is sufficiently to present $\left\langle\boldsymbol{S}_{q f}\right\rangle_{t}$ as a sum of constant term $\left\langle\boldsymbol{S}_{q f}\right\rangle_{0}$ (mean equilibrium value, calculated in MFA) and time dependent small deviation $\delta\left\langle\boldsymbol{S}_{q f}\right\rangle_{t}$ :

$$
\left\langle\boldsymbol{S}_{q f}\right\rangle_{t}=\left\langle\boldsymbol{S}_{q f}\right\rangle_{0}+\delta\left\langle\boldsymbol{S}_{q f}\right\rangle_{t} .
$$

\footnotetext{
${ }^{4}$ Sometimes one writes third term $-\frac{\hbar}{T_{2}}\left\langle\boldsymbol{S}_{q f}\right\rangle_{t \perp}$, describing decay process of the transverse component of pseudospin $\left\langle\boldsymbol{S}_{q f}\right\rangle_{t \perp}$, though it can be shown (Levitskii, Andrusyk \& Zachek, 2010) that its impact on Rochelle salt dynamics is negligible.
} 
Similarly:

$$
\mathcal{H}_{q f}(t)=\mathcal{H}_{f}^{(0)}+\delta \mathcal{H}_{q f}(t), \quad \overline{\left\langle\boldsymbol{S}_{q f}\right\rangle_{t}}=\left\langle\boldsymbol{S}_{q f}\right\rangle_{0}+\delta \overline{\left\langle\boldsymbol{S}_{q f}\right\rangle_{t}} .
$$

Now, we can linearize motion equation (32) by retaining terms, which are linear in deviations $\delta\left\langle\boldsymbol{S}_{q f}\right\rangle_{t}, \delta \mathcal{H}_{q f}(t), \delta \overline{\left\langle\boldsymbol{S}_{q f}\right\rangle_{t}}$ :

$$
\hbar \frac{d \delta\left\langle\boldsymbol{S}_{q f}\right\rangle_{t}}{d t}=\delta\left\langle\boldsymbol{S}_{q f}\right\rangle_{t} \times \mathcal{H}_{f}^{(0)}+\left\langle\boldsymbol{S}_{q f}\right\rangle_{0} \times \delta \mathcal{H}_{q f}(t)-\frac{\hbar}{T_{1}}\left[\delta\left\langle\boldsymbol{S}_{q f}\right\rangle_{t \|}-\delta \overline{\left\langle\boldsymbol{S}_{q f}\right\rangle_{t \|}}\right],
$$

where

$$
\begin{aligned}
& \delta \mathcal{H}_{q f}^{x}(t)=0, \quad \delta \mathcal{H}_{q f}^{y}(t)=0, \\
& \delta \mathcal{H}_{q 1}^{z}(t)=\sum_{q^{\prime}} J_{q q^{\prime}} \delta\left\langle S_{q^{\prime} 1}^{z}\right\rangle_{t}+\sum_{q^{\prime}} K_{q q^{\prime}} \delta\left\langle S_{q^{\prime} 2}^{z}\right\rangle_{t}-2 \psi_{14} \delta \varepsilon_{4 q}(t)+\mu \delta E_{1 q}(t), \\
& \delta \mathcal{H}_{q 2}^{z}(t)=\sum_{q^{\prime}} K_{q q^{\prime}} \delta\left\langle S_{q^{\prime} 1}^{z}\right\rangle_{t}+\sum_{q^{\prime}} J_{q q^{\prime}} \delta\left\langle S_{q^{\prime} 2}^{z}\right\rangle_{t}-2 \psi_{14} \delta \varepsilon_{4 q}(t)+\mu \delta E_{1 q}(t) .
\end{aligned}
$$

Now, we transform equation (38) into a form involving single variable $\delta\left\langle\boldsymbol{S}_{q f}\right\rangle_{t}$, then Fourier transform into the frequency domain and Fourier transform to k-space, ${ }^{5}$ introduce new theory parameters

$$
\tilde{R}_{k}^{+}=\frac{\tilde{J}_{k}+\tilde{K}_{k}}{2}, \quad \tilde{R}_{k}^{-}=\frac{\tilde{J}_{k}-\tilde{K}_{k}}{2} \quad\left(\tilde{J}_{k}=J_{k} / k_{B}, \quad \tilde{K}_{k}=K_{k} / k_{B}\right)
$$

and introduce new variables $\delta \boldsymbol{\xi}_{k}(t), \delta \boldsymbol{\sigma}_{k}(t)$

$$
\delta\left\langle\boldsymbol{S}_{k 1}\right\rangle_{t}=\frac{\delta \boldsymbol{\xi}_{k}(t)+\delta \boldsymbol{\sigma}_{k}(t)}{2}, \quad \delta\left\langle\boldsymbol{S}_{k 2}\right\rangle_{t}=\frac{\delta \boldsymbol{\xi}_{k}(t)-\delta \boldsymbol{\sigma}_{k}(t)}{2} .
$$

Upon application of these transformations, the Bloch equation (38) reduces to system of linear differential equations of the following matrix form:

$$
\left(A_{k}-\mathrm{i} \frac{\hbar}{k_{B}} \omega \cdot I\right) \delta x_{k}(\omega)=\left(\tilde{\mu} \delta E_{1 k}(\omega)-2 \tilde{\psi}_{14} \delta \varepsilon_{4 k}(\omega)\right) b .
$$

\footnotetext{
${ }^{5}$ Fourier transform to k-space is

$$
A_{q}=\sum_{k} a_{k} \exp (\mathrm{i} k q), \quad a_{k}=\frac{1}{N} \sum_{q} A_{q} \exp (-\mathrm{i} k q)
$$

for values dependent on $q$ (like $\delta\left\langle S_{q 1}^{z}\right\rangle_{t}$ and others) and

$$
M_{q_{1} q_{2}}=\frac{1}{N} \sum_{k} m_{k} \exp \left(\mathrm{i} k\left(q_{1}-q_{2}\right)\right), \quad m_{k}=\sum_{q_{2}} M_{q_{1} q_{2}} \exp \left(-\mathrm{i} k\left(q_{1}-q_{2}\right)\right) .
$$

for interaction constants dependent on $q_{1}$ and $q_{2}$ (like $J_{q q^{\prime}}$ and others). Here the dependency of $M_{q_{1} q_{2}}$ on difference $q_{1}-q_{2}$ was used.
} 
The following notations are used in this equation: $I$ is identity matrix, $\mathrm{i}$ is the imaginary unit,

$$
A_{k}=\left(\begin{array}{cccccc}
a_{11} & a_{12} & -\tilde{\Omega} & 0 & a_{15} & a_{16} \\
a_{21} & a_{22} & 0 & -\tilde{\Omega} & a_{16} & a_{15} \\
a_{31} & a_{32} & 0 & 0 & a_{35} & a_{36} \\
a_{41} & a_{42} & 0 & 0 & a_{36} & a_{35} \\
a_{51} & a_{52} & -a_{35} & -a_{36} & a_{55} & a_{56} \\
a_{61} & a_{62} & -a_{36} & -a_{35} & a_{56} & a_{55}
\end{array}\right), \delta x_{k}(\omega)=\left(\begin{array}{c}
\delta \xi_{k}^{z}(\omega) \\
\delta \sigma_{k}^{z}(\omega) \\
\delta \xi_{k}^{y}(\omega) \\
\delta \sigma_{k}^{y}(\omega) \\
\delta \xi_{k}^{x}(\omega) \\
\delta \sigma_{k}^{x}(\omega)
\end{array}\right), \quad b=\left(\begin{array}{l}
b_{1} \\
b_{2} \\
\xi^{x} \\
\sigma^{x} \\
b_{5} \\
b_{6}
\end{array}\right) .
$$

Matrix $A_{k}$ components:

$$
\begin{array}{ll}
a_{11}=U_{1}+\tilde{R}_{k}^{+} G_{1}, & a_{12}=U_{2}+\tilde{R}_{k}^{-} G_{2}, \quad a_{15}=V_{1}, \quad a_{16}=V_{2}, \\
a_{21}=U_{2}+\tilde{R}_{k}^{+} G_{2}, & a_{22}=U_{1}+\tilde{R}_{k}^{-} G_{1}, \\
a_{31}=\tilde{\Omega}-\tilde{R}_{k}^{+} \xi^{x}, & a_{32}=-\tilde{R}_{k}^{-} \sigma^{x}, a_{35}=-\left[\tilde{R}_{0}^{+} \xi^{z}-2 \tilde{\psi}_{14} \varepsilon_{4}\right], a_{36}=-\left[\tilde{R}_{0}^{-} \sigma^{z}+\tilde{\Delta}\right], \\
a_{41}=-\tilde{R}_{k}^{+} \sigma^{x}, & a_{42}=\tilde{\Omega}-\tilde{R}_{k}^{-} \xi^{x}, \\
a_{51}=V_{1}+\tilde{R}_{k}^{+} H_{1}, & a_{52}=V_{2}+\tilde{R}_{k}^{-} H_{2}, \quad a_{55}=W_{1}, \quad a_{56}=W_{2}, \\
a_{61}=V_{2}+\tilde{R}_{k}^{+} H_{2}, & a_{62}=V_{1}+\tilde{R}_{k}^{-} H_{1} ;
\end{array}
$$

components of vector $b$ :

$$
b_{1}=-G_{1}, \quad b_{2}=-G_{2}, \quad b_{5}=-H_{1}, \quad b_{6}=-H_{2},
$$

where following notations were used

$$
\begin{aligned}
& U_{1,2}=-\frac{1}{2 T_{1}^{\prime}}\left(\frac{\tilde{\varepsilon}_{1}^{2}}{\tilde{\lambda}_{1}^{2}} \pm \frac{\tilde{\varepsilon}_{2}^{2}}{\tilde{\lambda}_{2}^{2}}\right), \quad V_{1,2}=-\frac{1}{2 T_{1}^{\prime}}\left(\frac{\tilde{\Omega} \tilde{\varepsilon}_{1}}{\tilde{\lambda}_{1}^{2}} \pm \frac{\tilde{\Omega} \tilde{\varepsilon}_{2}}{\tilde{\lambda}_{2}^{2}}\right), \quad G_{1,2}=K_{1} \frac{\tilde{\varepsilon}_{1}^{2}}{\tilde{\lambda}_{1}^{2}} \pm K_{2} \frac{\tilde{\varepsilon}_{2}^{2}}{\tilde{\lambda}_{2}^{2}}, \\
& W_{1,2}=-\frac{1}{2 T_{1}^{\prime}}\left(\frac{\tilde{\Omega}^{2}}{\tilde{\lambda}_{1}^{2}} \pm \frac{\tilde{\Omega}^{2}}{\tilde{\lambda}_{2}^{2}}\right), \quad H_{1,2}=K_{1} \frac{\tilde{\Omega} \tilde{\varepsilon}_{1}}{\tilde{\lambda}_{1}^{2}} \pm K_{2} \frac{\tilde{\Omega} \tilde{\varepsilon}_{2}}{\tilde{\lambda}_{2}^{2}}, \quad K_{1,2}=\frac{1}{T_{1}^{\prime}} \frac{1}{4 T \cosh ^{2} \frac{\tilde{\lambda}_{1,2}}{2 T}},
\end{aligned}
$$

and relaxation time $T_{1}^{\prime}$ is

$$
T_{1}^{\prime}=\frac{k_{B}}{\hbar} T_{1}
$$

It is convenient to present the solution of Eq. (41) in a form

$$
\delta x_{k}(\omega)=\left(\tilde{\mu} \delta E_{1 k}(\omega)-2 \tilde{\psi}_{14} \delta \varepsilon_{4 k}(\omega)\right)\left[\left(A_{k}-\mathrm{i} \frac{\hbar}{k_{B}} \omega \cdot I\right)^{-1} b\right],
$$

where we denote the inverse of matrix $\left(A_{k}-\mathrm{i} \frac{\hbar}{k_{B}} \omega \cdot I\right)$ by $\left(A_{k}-\mathrm{i} \frac{\hbar}{k_{B}} \omega \cdot I\right)^{-1}$.

Now it is useful to present all variables in Eq. (21b) as a sum of constant (equilibrium) term and small variation term. The result for $\delta P_{1 k}(\omega)$ is

$$
\delta P_{1 k}(\omega)=e_{14}^{0} \delta \varepsilon_{4 k}(\omega)+\chi_{11}^{\varepsilon 0} \cdot \delta E_{1 k}(\omega)+\frac{\tilde{\mu}}{\tilde{v}} \cdot \delta \xi_{k}^{z}(\omega)
$$


If to note that $\delta \xi_{k}^{z}(\omega)$ is the first component of vector $\delta x_{k}(\omega)$, variation of polarization can be presented as

$$
\delta P_{1 k}(\omega)=e_{14}(\boldsymbol{k}, \omega) \delta \varepsilon_{4 k}(\omega)+\chi_{11}^{\varepsilon}(\boldsymbol{k}, \omega) \delta E_{1 k}(\omega) .
$$

Here $\chi_{11}^{\varepsilon}(\boldsymbol{k}, \omega)$ is dynamic susceptibility of a clamped crystal $\left(\delta \varepsilon_{4 k}(\omega)=0\right)$, and $e_{14}(\boldsymbol{k}, \omega)$ is dynamic coefficient of the piezoelectric stress:

$$
\chi_{11}^{\varepsilon}(\boldsymbol{k}, \omega)=\chi_{11}^{\varepsilon 0}+\frac{\tilde{\mu}^{2}}{\tilde{v}} F_{1}(\boldsymbol{k}, \mathrm{i} \omega), \quad e_{14}(\boldsymbol{k}, \omega)=e_{14}^{0}-\frac{2 \tilde{\psi}_{14} \tilde{\mu}}{\tilde{v}} F_{1}(\boldsymbol{k}, \mathrm{i} \omega)
$$

The following notation was used:

$$
F_{1}(\boldsymbol{k}, \mathrm{i} \omega)=\left[\left(A_{k}-\mathrm{i} \frac{\hbar}{k_{B}} \omega \cdot I\right)^{-1} b\right]_{1}^{\prime}
$$

where subscript ' 1 ' denotes the first component of the vector derived by multiplication of matrix $\left(A_{k}-\mathrm{i} \frac{\hbar}{k_{B}} \omega \cdot I\right)^{-1}$ and vector $b$.

Analysis shows that $F_{1}(\mathbf{0}, 0)=f_{1}\left(T, \sigma_{4}, E_{1}\right)$ at any relaxation times $T_{1}$. Therefore, all dynamic physical characteristics are equal to correspondent static characteristics at $\omega=0$.

The best agreement between theory and experiment for RS is reached at (Levitskii, Andrusyk \& Zachek, 2010)

$$
T_{1}=1.767 \times 10^{-13} \mathrm{~s} \quad\left(T_{1}^{\prime}=2.313 \times 10^{-2} \mathrm{~K}\right) .
$$

It is easily seen that the function $F_{1}(\mathbf{k}, \mathrm{i} \omega)$ is a rational function of $\mathrm{i} \omega$, where numerator is polynomial function of degree not higher than 5 and denominator is polynomial function of degree 6 . Therefore, we can decompose function $F_{1}(\mathbf{k}, \mathbf{i} \omega)$ into partial fractions:

$$
F_{1}(\boldsymbol{k}, \mathrm{i} \omega)=\sum_{i=1}^{n} \frac{k_{i} \tau_{i}}{1+\mathrm{i} \omega \tau_{i}}+\sum_{j=1}^{m} \frac{M_{j}(\mathrm{i} \omega)+N_{j}}{(\mathrm{i} \omega)^{2}+p_{j}(\mathrm{i} \omega)+q_{j}} .
$$

Here coefficients $k_{i}, \tau_{i}, M_{j}, N_{j}, p_{j}, q_{j}$ are real numbers, $n$ is number of real (equal to $-1 / \tau_{i}$ ) eigen values and $2 m$ is number of complex eigen values of matrix $A_{k}$. Values $n$ and $2 m$ are defined by theory parameters and temperature. However, matrix $A_{k}$ has 6 eigen values in total. The first sum in Eq. (52) is a contribution of Debye (relaxation) modes into order parameter dynamics, and the second sum is a contribution of resonance modes. In RS case we have $n=2, m=2$ at all temperatures.

The results of calculations performed in the center of the Brillouin zone $(\boldsymbol{k}=0)$ are presented below. Fig. 5 presents frequency dependencies of dielectric permittivity of clamped crystal in dispersion region $\left(10^{9} \mathrm{~Hz}-10^{11} \mathrm{~Hz}\right)$ calculated theoretically along with experimental data. As figure shows, Mitsui model is able to describe dielectric permittivity in dispersion region.

The correspondence between theory and experimental data for dynamic dielectric permittivity deserves special attention. Methods for experimental measurements of dynamic dielectric permittivity does not allow to assert that namely clamped crystal permittivity was 

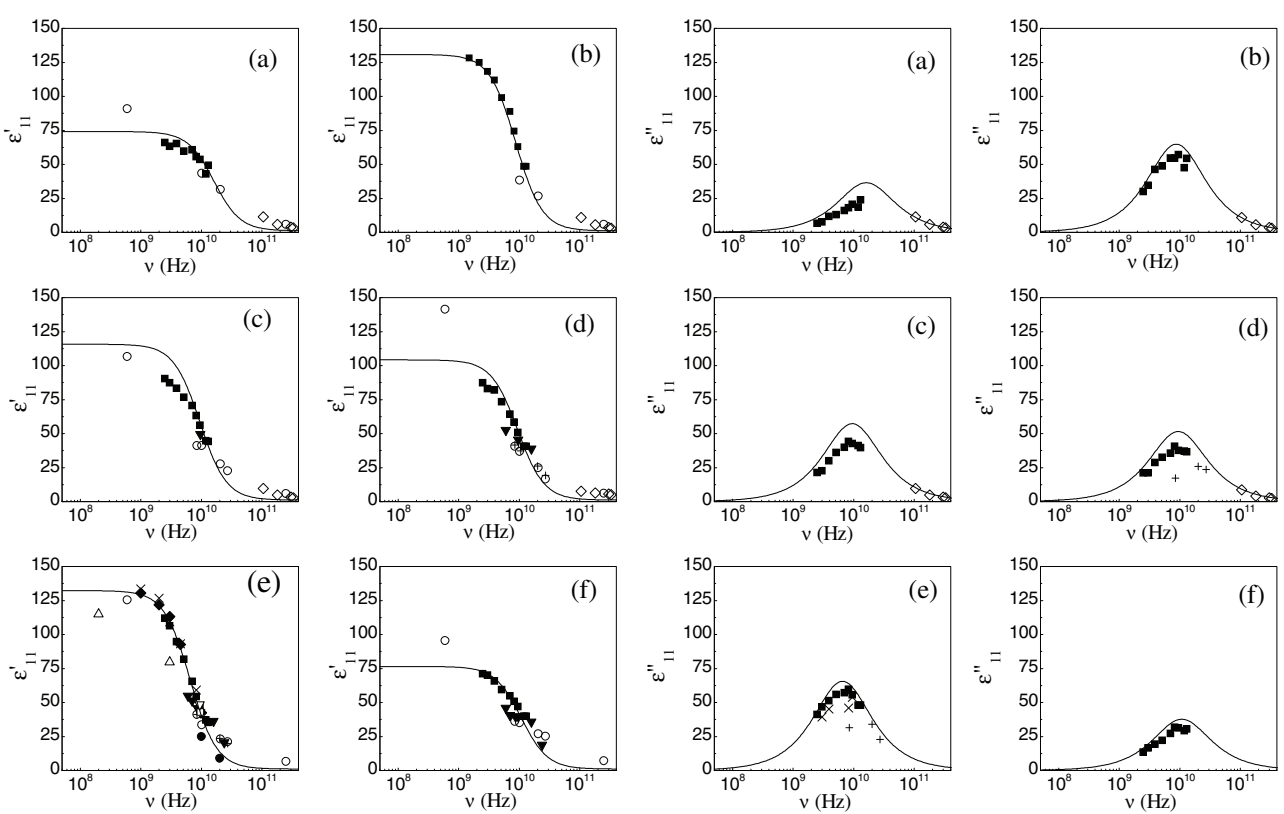

Fig. 5. The frequency dependence of the real and imaginary part of dielectric permittivity, calculated theoretically (lines) at different temperatures $T$ (K): (a) 235, (b) 245, (c) 265, (d) 285, (e) 305, (f) 315. Points represent experimental data: ( (Sandy \& Jones, 1968), ○ (Poplavko et al., 1974), + (Pereverzeva, 1974), $\mathbf{\nabla}$ (Deyda, 1967), • (Akao \& Sasaki, 1955), (Müser \& Potthaest, 1967), × (Kołodziej, 1975), $\diamond($ Volkov et al., 1980), $\triangle($ Sandy \& Jones, 1968), $\nabla$ (Jäckle, 1960).

measured. However, in the next subsection it will be demonstrated that frequency clamping of a crystal occurs in microwave region and the experimental data for dielectric permittivity in this region correspond specifically to theoretically calculated dielectric permittivity of a clamped crystal

Analysis testifies that the contribution of one relaxation mode constitutes more than $99 \%$ of the total permittivity along the whole temperature range at frequencies $10^{8} \mathrm{~Hz}-10^{12} \mathrm{~Hz}$. This mode is responsible for ferroelectric instability. Thus, dynamics of RS within microwave region is of Debye relaxation type. The same conclusion was derived within the model without transverse field (Levitskii et al., 2003).

Temperature dependencies of relaxation time of the mode responsible for ferroelectric instability obtained here and correspondent experimental data are presented in Fig. 6. As this figure shows the model with the piezoelectric coupling successfully solves the problem encountered by the conventional theories - incorrect temperature dependence of relaxation times near the Curie points. Theoretical temperature curve of $\tau^{-1}(\mathrm{~T})$ obtained here has two finite minima at the transition points, as opposed to vanishing of the inverse relaxation time obtained within the Mitsui model without piezoelectric coupling (Žekš et al., 1971). 


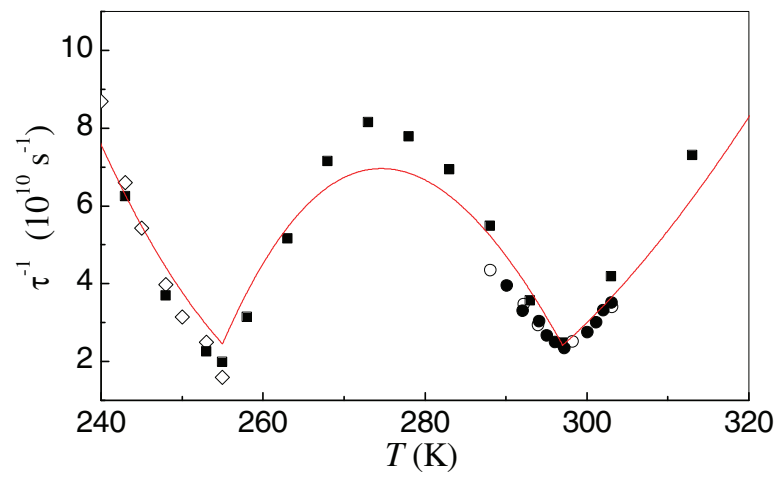

Fig. 6. Dependency of inverse relaxation times on temperature. Solid line presents the result

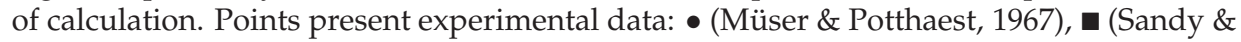
Jones, 1968), ○ (Kołodziej, 1975), $\diamond($ Volkov et al., 1980).

\subsection{Dynamics of deformation. Dielectric susceptibility of a free crystal}

We shall now give detailed consideration to dynamic dielectric response of a free crystal. Expression for the stress variation is obtained in a similar way to Eq. (48)

$$
\delta \sigma_{4 k}(\omega)=c_{44}(\boldsymbol{k}, \omega) \delta \varepsilon_{4 k}(\omega)-e_{14}(\boldsymbol{k}, \omega) \delta E_{1 k}(\omega),
$$

where

$$
c_{44}(\boldsymbol{k}, \omega)=c_{44}^{E 0}-\frac{4 \tilde{\psi}_{14}^{2}}{\tilde{v}} F_{1}(\boldsymbol{k}, \mathrm{i} \omega)
$$

and $e_{14}(\boldsymbol{k}, \omega)$ is presented in Eq. (49).

Expressing Eq. (53) $\delta \varepsilon_{4 k}(\omega)$ through $\delta \sigma_{4 k}(\omega)$ and taking account of Eq. (48) we can write:

$$
\delta P_{1 k}(\omega)=d_{14}(\boldsymbol{k}, \omega) \delta \sigma_{4 k}(\omega)+\chi_{11}^{\sigma}(\boldsymbol{k}, \omega) \delta E_{1 k}(\omega),
$$

where

$$
d_{14}(\boldsymbol{k}, \omega)=\frac{e_{14}(\boldsymbol{k}, \omega)}{c_{44}(\boldsymbol{k}, \omega)}, \quad \chi_{11}^{\sigma}(\boldsymbol{k}, \omega)=\chi_{11}^{\varepsilon}(\boldsymbol{k}, \omega)+e_{14}(\boldsymbol{k}, \omega) d_{14}(\boldsymbol{k}, \omega) .
$$

It is well to bear in mind that value $\chi_{11}^{\sigma}(\boldsymbol{k}, \omega)$ is not free crystal permittivity. For the crystal not affected by external mechanical stress the condition $\delta \sigma_{4 q}=0$ is true for all sites on the crystal surface, while all the internal stresses and strains are determined by Newtonian equations. Thus, to study dynamic dielectric response of a free crystal, in addition to the study of pseudospin system response it is needed to consider the dynamic deformation response of a crystal lattice caused by piezoelectric coupling. We will describe internal stresses and strains dynamics by Newtonian equations of motion for continuum (Authier, 2003, chap. 1.3). This approach is justified by virtue of the fact that, as we will see, in the frequency range under the study $\left(10^{4} \mathrm{~Hz}-10^{7} \mathrm{~Hz}\right)$, characteristic length of stress and strain change in the crystal is much larger than unit cell size.

We will have to join Eq. (55) obtained for discrete medium with the equations describing the dynamics of deformations, which are the continuum equations. This could be done by considering that physical characteristics $A_{k}(\omega)$ (polarization, stress and electric field) change 
little within the unit cell. In our case this condition is satisfied very well. So, we can write:

$$
A_{k}(\omega)=\frac{1}{N} \sum_{q} a_{q} \exp (-\mathrm{i} \boldsymbol{k q}) \simeq \frac{1}{V} \int_{V} a(\boldsymbol{r}) \exp (-\mathrm{i} \boldsymbol{k} \boldsymbol{r}) \mathrm{d} \boldsymbol{r} .
$$

For simplicity we will assume that physical coefficients are not dependent on wavevector:

$$
c_{44}(\boldsymbol{k}, \omega)=c_{44}(\omega), \quad e_{14}(\boldsymbol{k}, \omega)=e_{14}(\omega), \quad \chi_{11}^{\varepsilon, \sigma}(\boldsymbol{k}, \omega)=\chi_{11}^{\varepsilon, \sigma}(\omega)
$$

Let us consider the equation describing the dynamics of deformations of a thin rectangular plate $a \times b$ of Rochelle salt crystal cut in the (100) plane (X cut) (specific numerical calculations will be made for the crystal plate of $1 \times 1 \mathrm{~cm}^{2}$ ):

$$
\rho \frac{\partial^{2} u_{i}}{\partial t^{2}}=\sum_{j} \frac{\partial \sigma_{i j}}{\partial x_{j}}
$$

Here $\rho=1.767 \mathrm{~g} / \mathrm{cm}^{3}$ is crystal density (we suppose it is not temperature dependent), $u_{i}$ are components of the displacement vector, and $\sigma_{i j}$ are components of the stress tensor. Similarly to above, we decompose all physical characteristics in Eq. (59) into the sum of equilibrium static part and small deviation. We also assume that oscillating process occurs in $y z$-plane. It means that all values are uniformed along $x$-axes and $u_{x}=0$. Then system of equations (59) is reduced to

$$
\left.\begin{array}{l}
-\rho \omega^{2} \delta u_{y}=\frac{\partial \delta \sigma_{y y}}{\partial y}+\frac{\partial \delta \sigma_{y z}}{\partial z} \\
-\rho \omega^{2} \delta u_{z}=\frac{\partial \delta \sigma_{y z}}{\partial y}+\frac{\partial \delta \sigma_{z z}}{\partial z}
\end{array}\right\}
$$

where equations are already Fourier transformed into the frequency domain.

Strain tensor variations can be expressed in terms of displacements:

$$
\delta \varepsilon_{i j}=\frac{1}{2}\left(\frac{\partial \delta u_{i}}{\partial x_{j}}+\frac{\partial \delta u_{j}}{\partial x_{i}}\right) .
$$

By differentiating system (60) we can transform it to the form with unknowns $\delta \varepsilon_{\alpha}$ :

$$
\begin{aligned}
& -\rho \omega^{2} \delta \varepsilon_{2}=\frac{\partial^{2} \delta \sigma_{2}}{\partial y^{2}}+\frac{\partial^{2} \delta \sigma_{4}}{\partial y \partial z} \\
& -\rho \omega^{2} \delta \varepsilon_{3}=\frac{\partial^{2} \delta \sigma_{4}}{\partial y \partial z}+\frac{\partial^{2} \delta \sigma_{3}}{\partial z^{2}} \\
& -\rho \omega^{2} \delta \varepsilon_{4}=\frac{\partial^{2} \delta \sigma_{2}}{\partial y \partial z}+\frac{\partial^{2} \delta \sigma_{3}}{\partial y \partial z}+\left(\frac{\partial^{2}}{\partial y^{2}}+\frac{\partial^{2}}{\partial z^{2}}\right) \delta \sigma_{4}
\end{aligned}
$$

where Voigt's one-index notations and correspondence between strains in tensor and Voigt's notations $\delta \varepsilon_{2}=\delta \varepsilon_{y y}, \delta \varepsilon_{3}=\delta \varepsilon_{z z}, \delta \varepsilon_{4}=2 \delta \varepsilon_{y z}$ were used. 
Considering the form of elastic tensor Eq. (1) and tensor of piezoelectric stress Eq. (2) we can write:

$$
\begin{aligned}
& \delta \sigma_{2}=c_{22} \delta \varepsilon_{2}+c_{23} \delta \varepsilon_{3}+c_{24} \delta \varepsilon_{2}-e_{12} \delta E_{1} \\
& \delta \sigma_{3}=c_{23} \delta \varepsilon_{2}+c_{33} \delta \varepsilon_{3}+c_{34} \delta \varepsilon_{4}-e_{13} \delta E_{1} \\
& \delta \sigma_{4}=c_{24} \delta \varepsilon_{2}+c_{34} \delta \varepsilon_{3}+c_{44} \delta \varepsilon_{4}-e_{14} \delta E_{1} .
\end{aligned}
$$

All stresses and strains are functions of both frequency and coordinates. Elastic constants depend on frequency and are coordinates-independent (resulting from Eq. (58)).

We are interested in effects occurring at frequencies $10^{4} \mathrm{~Hz}-10^{8} \mathrm{~Hz}$, that is why for the crystal plate $1 \times 1 \mathrm{~cm}^{2}$ the field $\delta E_{1}$ can be considered as homogeneous. Under these conditions, the system Eq. (62) can be rewritten as:

$$
\begin{aligned}
-\rho \omega^{2} \delta \varepsilon_{2}= & c_{22} \frac{\partial^{2} \delta \varepsilon_{2}}{\partial y^{2}}+c_{23} \frac{\partial^{2} \delta \varepsilon_{3}}{\partial y^{2}}+c_{24} \frac{\partial^{2} \delta \varepsilon_{4}}{\partial y^{2}}+c_{24} \frac{\partial^{2} \delta \varepsilon_{2}}{\partial y \partial z}+c_{34} \frac{\partial^{2} \delta \varepsilon_{3}}{\partial y \partial z}+c_{44} \frac{\partial^{2} \delta \varepsilon_{4}}{\partial y \partial z} \\
-\rho \omega^{2} \delta \varepsilon_{3}= & c_{23} \frac{\partial^{2} \delta \varepsilon_{2}}{\partial z^{2}}+c_{33} \frac{\partial^{2} \delta \varepsilon_{3}}{\partial z^{2}}+c_{34} \frac{\partial^{2} \delta \varepsilon_{4}}{\partial z^{2}}+c_{24} \frac{\partial^{2} \delta \varepsilon_{2}}{\partial y \partial z}+c_{34} \frac{\partial^{2} \delta \varepsilon_{3}}{\partial y \partial z}+c_{44} \frac{\partial^{2} \delta \varepsilon_{4}}{\partial y \partial z} \\
-\rho \omega^{2} \delta \varepsilon_{4}= & \left(c_{22}+c_{23}\right) \frac{\partial^{2} \delta \varepsilon_{2}}{\partial y \partial z}+\left(c_{23}+c_{33}\right) \frac{\partial^{2} \delta \varepsilon_{3}}{\partial y \partial z}+\left(c_{24}+c_{34}\right) \frac{\partial^{2} \delta \varepsilon_{4}}{\partial y \partial z}+c_{24} \Delta \delta \varepsilon_{2} \\
& +c_{34} \Delta \delta \varepsilon_{3}+c_{44} \Delta \delta \varepsilon_{4}
\end{aligned}
$$

where $\Delta \equiv\left(\frac{\partial^{2}}{\partial y^{2}}+\frac{\partial^{2}}{\partial z^{2}}\right)$.

This system of equations should be supplemented by boundary conditions, which imply that stress is equal to zero on the crystal boundaries:

$$
\begin{aligned}
& \left.\left(c_{22} \delta \varepsilon_{2}+c_{23} \delta \varepsilon_{3}+c_{24} \delta \varepsilon_{4}-e_{12} \delta E_{1}\right)\right|_{\Sigma}=0 \\
& \left.\left(c_{23} \delta \varepsilon_{2}+c_{33} \delta \varepsilon_{3}+c_{34} \delta \varepsilon_{4}-e_{13} \delta E_{1}\right)\right|_{\Sigma}=0 \\
& \left.\left(c_{24} \delta \varepsilon_{2}+c_{34} \delta \varepsilon_{3}+c_{44} \delta \varepsilon_{4}-e_{14} \delta E_{1}\right)\right|_{\Sigma}=0
\end{aligned}
$$

where $\Sigma$ is a rectangle with sides $a \times b$ in $y z$-plane. This system is a closed equation system and allows one to describe the dynamics of deformation of a Rochelle salt crystal. This system of equations and its boundary conditions are of a simpler form in paraelectric phases:

$$
\begin{aligned}
& -\rho \omega^{2} \delta \varepsilon_{2}=c_{22} \frac{\partial^{2} \delta \varepsilon_{2}}{\partial y^{2}}+c_{23} \frac{\partial^{2} \delta \varepsilon_{3}}{\partial y^{2}}+c_{44} \frac{\partial^{2} \delta \varepsilon_{4}}{\partial y \partial z} \\
& -\rho \omega^{2} \delta \varepsilon_{3}=c_{23} \frac{\partial^{2} \delta \varepsilon_{2}}{\partial z^{2}}+c_{33} \frac{\partial^{2} \delta \varepsilon_{3}}{\partial z^{2}}+c_{44} \frac{\partial^{2} \delta \varepsilon_{4}}{\partial y \partial z} \\
& -\rho \omega^{2} \delta \varepsilon_{4}=\left(c_{22}+c_{23}\right) \frac{\partial^{2} \delta \varepsilon_{2}}{\partial y \partial z}+\left(c_{23}+c_{33}\right) \frac{\partial^{2} \delta \varepsilon_{3}}{\partial y \partial z}+c_{44} \Delta \delta \varepsilon_{4},
\end{aligned}
$$




$$
\begin{aligned}
& \left.\left(c_{22} \delta \varepsilon_{2}+c_{23} \delta \varepsilon_{3}\right)\right|_{\Sigma}=0 \\
& \left.\left(c_{23} \delta \varepsilon_{2}+c_{33} \delta \varepsilon_{3}\right)\right|_{\Sigma}=0 \\
& \left.\left(c_{44} \delta \varepsilon_{4}-e_{14} \delta E_{1}\right)\right|_{\Sigma}=0 .
\end{aligned}
$$

One can proceed with further simplifications. Consider that elastic constants $c_{22}, c_{23}, c_{33}$ are of the same order and are much larger than $c_{44}$ (see Fig. 1), especially in the vicinity of a critical point. Then $\delta \varepsilon_{2}$ and $\delta \varepsilon_{3}$ become small compared to $\delta \varepsilon_{4}$ and can be treated as zero. Therefore, system Eq. (66) and boundary conditions Eq. (67) reduce to

$$
\begin{aligned}
& \Delta \delta \varepsilon_{4}+\frac{\rho \omega^{2}}{c_{44}} \delta \varepsilon_{4}=0 \\
& \left.\delta \varepsilon_{4}\right|_{\Sigma}=\frac{e_{14}}{c_{44}} \delta E_{1} .
\end{aligned}
$$

Similarly, equation (68) can be obtained for ferroelectric phase.

It is noteworthy that in paraelectric phase in case of small $c_{22}, c_{23}, c_{33}$ we would receive equation (68) again. However, in this case strains $\delta \varepsilon_{2}$ and $\delta \varepsilon_{3}$ are not equal to zero:

$$
\delta \varepsilon_{2}=\delta \varepsilon_{3}=-\frac{c_{44}}{\rho \omega^{2}} \frac{\partial^{2} \delta \varepsilon_{4}}{\partial y \partial z}
$$

It is more convenient to solve equation 68 by rewriting it in terms of the variation of stress. Taking into account Eq. (53), from which

$$
\delta \varepsilon_{4}(\omega)=\frac{1}{c_{44}(\omega)} \delta \sigma_{4}(\omega)+\frac{e_{14}(\omega)}{c_{44}(\omega)} \delta E_{1}(\omega)
$$

and taking into account field homogeneity we can write:

$$
\begin{aligned}
& \Delta \delta \sigma_{4}+\frac{\rho \omega^{2}}{c_{44}} \delta \sigma_{4}=-\frac{\rho \omega^{2}}{c_{44}} e_{14} \delta E_{1} \\
& \left.\delta \sigma_{4}\right|_{\Sigma}=0 .
\end{aligned}
$$

By introducing a new variable $u(\omega, y, z)$, where

$$
\delta \sigma_{4}=-u(\omega, y, z) \cdot e_{14}(\omega) \delta E_{1}(\omega)
$$

and considering Eq. (55) we obtain the expression for free crystal permittivity in the center of the Brillouin zone:

$$
\tilde{\chi}_{11}^{\sigma}(\omega)=\chi_{11}^{\sigma}(\omega)+N(\omega)\left(\chi_{11}^{\varepsilon}(\omega)-\chi_{11}^{\sigma}(\omega)\right),
$$

where

$$
N(\omega)=\frac{1}{a b} \int_{0}^{a} \int_{0}^{b} \mathrm{~d} y \mathrm{~d} z u(\omega, y, z),
$$


and $u(\omega, y, z)$ is a solution for

$$
\begin{aligned}
& \Delta u+\frac{\rho \omega^{2}}{c_{44}(\omega)} u=\frac{\rho \omega^{2}}{c_{44}(\omega)} \\
& \left.u\right|_{\Sigma}=0 .
\end{aligned}
$$

It is easy to see that in the limit of low and high frequencies one has:

$$
\begin{array}{ll}
\omega \rightarrow 0: & u(y, z) \rightarrow 0 \Longrightarrow N(\omega) \rightarrow 0 \Longrightarrow \tilde{\chi}_{11}^{\sigma}(\omega) \rightarrow \chi_{11}^{\sigma}(\omega) \\
\omega \rightarrow \infty: & u(y, z) \rightarrow 1 \Longrightarrow N(\omega) \rightarrow 1 \Longrightarrow \tilde{\chi}_{11}^{\sigma}(\omega) \rightarrow \chi_{11}^{\varepsilon}(\omega) .
\end{array}
$$

From Eq. (74) and Eq. (75) it is clear that $\chi_{11}^{\sigma}(\omega)$ would be the permittivity of free crystal in case of completely rigid material of crystal $\left(c_{44}(\omega)=\infty\right)$ or at its zero inertia $(\rho=0)$.

Eq. (74) is inhomogeneous Helmholtz equation with zero Dirichlet boundary conditions. It has the following solution: ${ }^{6}$

$$
\begin{aligned}
u(\omega, y, z)=\sum_{i, j=0}^{\infty} & \frac{16}{1-\frac{c_{44}(\omega)}{\rho \omega^{2}} \pi^{2}\left(\frac{(2 i+1)^{2}}{a^{2}}+\frac{(2 j+1)^{2}}{b^{2}}\right)} \cdot \frac{1}{\pi^{2}(2 i+1)(2 j+1)} \\
& \times \sin \frac{\pi(2 i+1) y}{a} \sin \frac{\pi(2 j+1) z}{b} .
\end{aligned}
$$

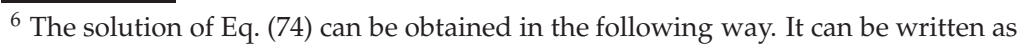

$$
u(y, z)=\sum_{k, n=1}^{\infty} \frac{\left(v, \psi_{k n}\right)}{\lambda_{k n}} \cdot \psi_{k n}(y, z)
$$

where $v \equiv \frac{\rho \omega^{2}}{c_{44}(\omega)},(f, g)$ denotes scalar product of functions $f(y, z)$ and $g(y, z)$ :

$$
(f, g)=\int_{\Sigma} f g \mathrm{~d} \Sigma
$$

$\psi_{k n}(y, z)$ and $\lambda_{k n}$ are eigenfunctions and eigenvalues of the Helmholtz operator $\Delta+v$ on a domain $\Sigma$ (rectangle $a \times b$ ):

$$
\begin{aligned}
& \psi_{k n}(y, z)=\frac{2}{\sqrt{a b}} \sin \frac{\pi k y}{a} \sin \frac{\pi n z}{b}, \\
& \lambda_{k n}=-\pi^{2}\left(\frac{k^{2}}{a^{2}}+\frac{n^{2}}{b^{2}}\right)+v,
\end{aligned}
$$

which is easily checked directly. Let us use the known fact that the system of eigenfunctions of a Hermitian operator (which is the Helmholtz operator) is a complete set of orthogonal functions. Therefore, any analytic function can be decomposed into a series of functions $\psi_{k m}$. For $v$ treated as a function the decomposition can be written

$$
v=\sum_{k, n} a_{k n} \psi_{k n}
$$

where

$$
a_{p q}=\left(v, \psi_{p q}\right),
$$

which is easy to obtain by calculating the scalar product of left and right parts of Eq. (77) and $\psi_{p q}$. Now, by substitution of Eq. (76) into Eq. (74) we see that Eq. (76) is the needed solution. 
After performing the integration we get $N(\omega)$ :

$$
N(\omega)=\sum_{i, j=0}^{\infty} \frac{64}{1-\frac{c_{44}(\omega)}{\rho \omega^{2}} \pi^{2}\left(\frac{(2 i+1)^{2}}{a^{2}}+\frac{(2 j+1)^{2}}{b^{2}}\right)} \cdot \frac{1}{\left[\pi^{2}(2 i+1)(2 j+1)\right]^{2}},
$$

where at small $\operatorname{Im}\left[c_{44}(\omega)\right]$ resonance frequencies are equal

$$
\omega_{i, j}=\sqrt{\frac{c_{44}(\omega)}{\rho}} \pi \sqrt{\frac{(2 i+1)^{2}}{a^{2}}+\frac{(2 j+1)^{2}}{b^{2}}} .
$$

Previously, for a square lattice the following resonance frequencies were obtained (Moina et al., 2005):

$$
\omega_{i}=\sqrt{\frac{c_{44}(\omega)}{\rho}} \pi \frac{(2 i+1)}{a},
$$

while our study has shown that resonance occurs at frequencies

$$
\omega_{i, j}=\sqrt{\frac{c_{44}(\omega)}{\rho}} \pi \sqrt{\frac{(2 i+1)^{2}+(2 j+1)^{2}}{a^{2}}} .
$$

In particular, for the first resonance frequency we obtained a value

$$
\omega_{0,0}=\frac{\pi}{a} \sqrt{\frac{2 c_{44}(\omega)}{\rho}}
$$

while the previous result claims

$$
\omega_{0}=\frac{\pi}{a} \sqrt{\frac{c_{44}(\omega)}{\rho}} .
$$

Besides, one can see that resonance frequencies derived here are more compact compared to the previous result. Unfortunately, we have not found appropriate experimental data that could verify which of the two is correct.

It should be noted that due to the presence of imaginary part in $c_{44}(\omega)$, resonance peaks of dielectric permittivity of free crystal do not have singularity. According to Eq. (80) when approaching a phase transition point, the frequency of the first resonance peak tends to zero. Analysis of Eq. (82) shows that in case of square plate resonance frequencies degeneracy $\omega_{i, j}=$ $\omega_{j, i}$ occurs. And in case of rectangular plate with $a$ and $b$, which are little different from each other, resonance spectrum consists of couples of close resonance frequencies $\omega_{i, j}$ and $\omega_{j, i}$.

Let us show the results of the calculation of dynamic permittivity of free crystal for a thin plate of $X$-cut Rochelle salt crystal of size $1 \times 1 \mathrm{~cm}^{2}$. Fig. 7 illustrates frequency dependence of dynamic permittivity in the paraelectric phase at $T=305 \mathrm{~K}$.

As it is illustrated in this figure, at $\omega \rightarrow 0$ we obtain static permittivity of free crystal. Below the first resonance peak, the dielectric permittivity almost coincides with the static permittivity of free crystal. In the range $10^{4} \mathrm{~Hz}-10^{8} \mathrm{~Hz}$ one gets numerous resonance peaks. Above the resonance range, crystal is 'clamped' by the frequency and at $10^{9} \mathrm{~Hz}-$ $10^{11} \mathrm{~Hz}$ for dynamic permittivity one gets susceptibility of clamped crystal, considered in 
previous subsection. Similar behaviour of dynamic permittivity of free crystal is observed in low-temperature paraelectric and ferroelectric phases.
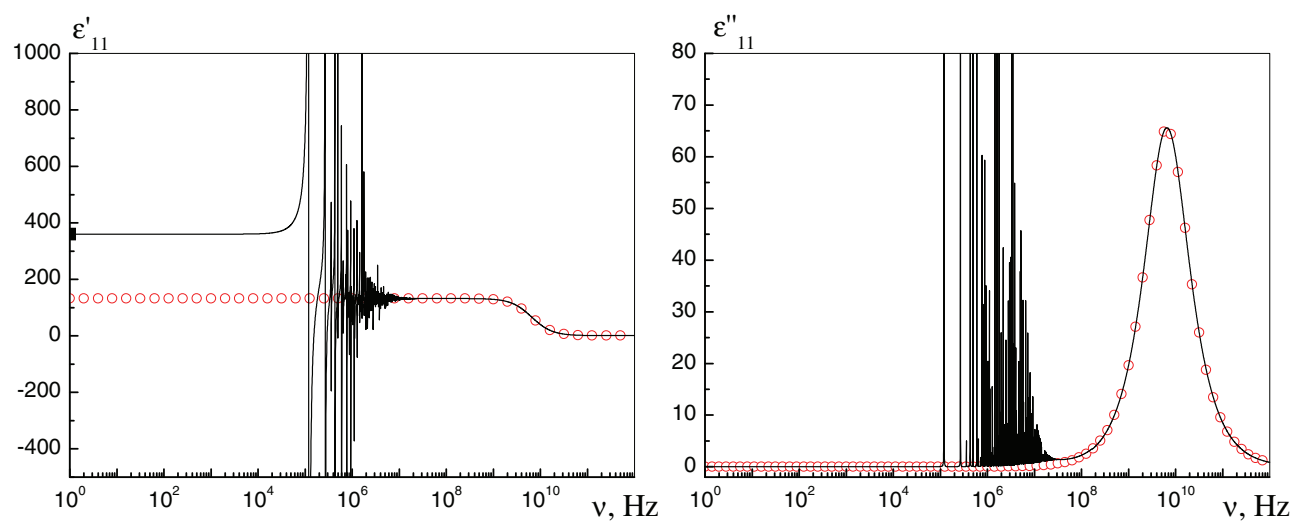

Fig. 7. Dynamic free crystal dielectric permittivity (solid line) at $T=305 \mathrm{~K}$. Points $\circ$ present clamped crystal dielectric permittivity at $T=305 \mathrm{~K}$. Point $\square$ presents static free crystal permittivity.

Figs. 8, 9, 10, 11 present distributions of absolute value, real part and imaginary part of function $u(y, z)$. White colour denotes small value, black colour denotes large value. Calculations were performed for square plate $1 \times 1 \mathrm{~cm}^{2}$ at $T=305 \mathrm{~K}$ and different frequencies. Internal stress is proportional to the function $u$ (see Eq. (71)), while according to Eq. (69) strain is

$$
\delta \varepsilon_{4}=(1-u(\omega, y, z)) \frac{e_{14}(\omega)}{c_{44}(\omega)} \delta E_{1}(\omega)
$$

As might be expected, at low frequency $\left(v=10^{5} \mathrm{~Hz}\right.$, Fig. 8) the amplitude of stress in the center of the plate is maximum and it gradually reduces to zero when approaching the plate edge. When increasing the frequency $\left(v=2.5 \times 10^{5} \mathrm{~Hz}\right.$ and $\left.v=5.95 \times 10^{5} \mathrm{~Hz}\right)$ the regions with large and small stress amplitude start alternating (Figs. 9 and 10). However, one can see that characteristic length of stress (strain) altering is much greater than lattice sizes and, hence, approach of continuum medium to considering of the deformation dynamics is justified. Upon further increase of frequency, the stress becomes uniformed throughout the crystal volume, but close to the crystal edge it slightly increases, and then is reduced to zero at the crystal boundary. At frequencies higher than the piezoelectric resonance frequencies the crystal is clamped by frequency and internal stress is defined as $\delta \sigma_{4}(\omega)=-e_{14}(\omega) \delta E_{1}(\omega)$. Crystal plate of $1 \times 1 \mathrm{~cm}^{2}$ can be considered as clamped at $T=305 \mathrm{~K} v=5 \times 10^{7} \mathrm{~Hz}$ according to Fig. 7. Distribution diagram of $u$ in Fig. 11 visualize phenomenon of crystal clamping.

\section{Concluding remarks}

In this chapter we considered piezoelectric effect in Rochelle salt. We based our study on the Mitsui model which explains the ferroelectric phase transition in Rochelle salt at microscopic level and is able to describe its thermodynamic and dynamic (dielectric relaxation) properties. Results obtained for the physical coefficients were used for study of piezoelectric resonance. It should be noted that the ratio between the elastic constants of Rochelle salt allowed to reduce 

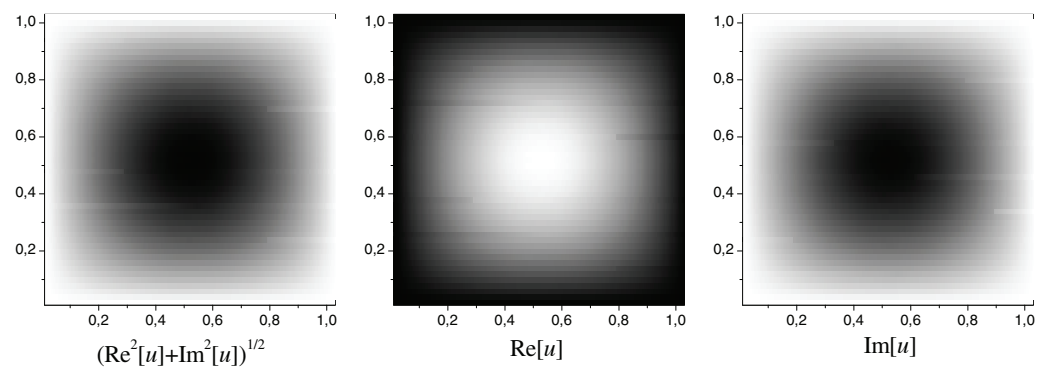

Fig. 8. Distributions of absolute value, real part and imaginary part of $u(y, z)$ at $v=10^{5} \mathrm{~Hz}$.
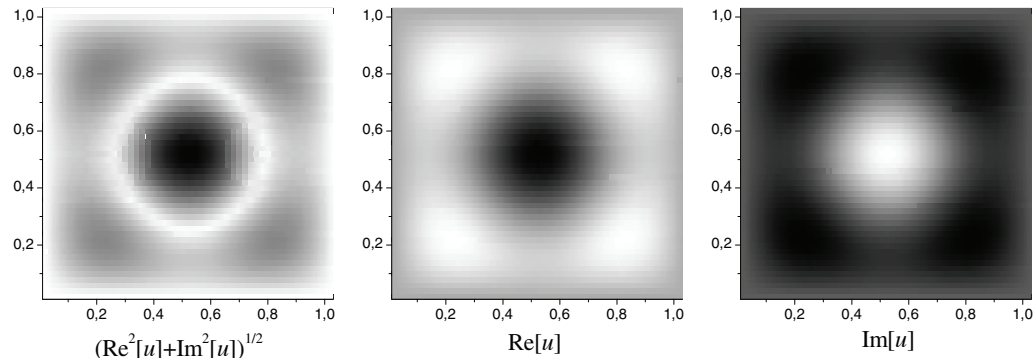

Fig. 9. Distributions of absolute value, real part and imaginary part of $u(y, z)$ at $v=2.6 \times 10^{5} \mathrm{~Hz}$.
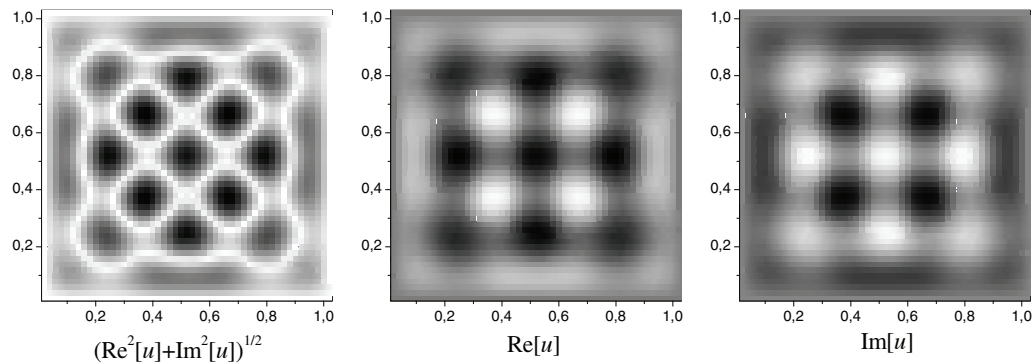

Fig. 10. Distributions of absolute value, real part and imaginary part of $u(y, z)$ at $v=5.95 \times 10^{5} \mathrm{~Hz}$.

the original system of equations for the dynamics of deformations to Helmholtz equation with known boundary condition. This simplification allowed to obtain the solution analytically. Analytical solution, in turn, allowed to obtain resonant frequencies and explicitly showed the phenomenon of frequency crystal clamping.

Nevertheless, the proposed approach has some drawbacks. Specifically, the dynamic strain change along $x$ axis was neglected. We assume that for a thin plate such neglect is justified, but this assumption should be confirmed by numerical calculations. Consideration of strain change along $x$ axis will make it possible not to be restricted by a thin plate.

Also, some important issues remain unexplored. In particular, the influence of ferroelectric phase transition on piezoelectric resonance remains open. The study of this issue requires to consider the system Eq. (64), which can be performed only numerically. 

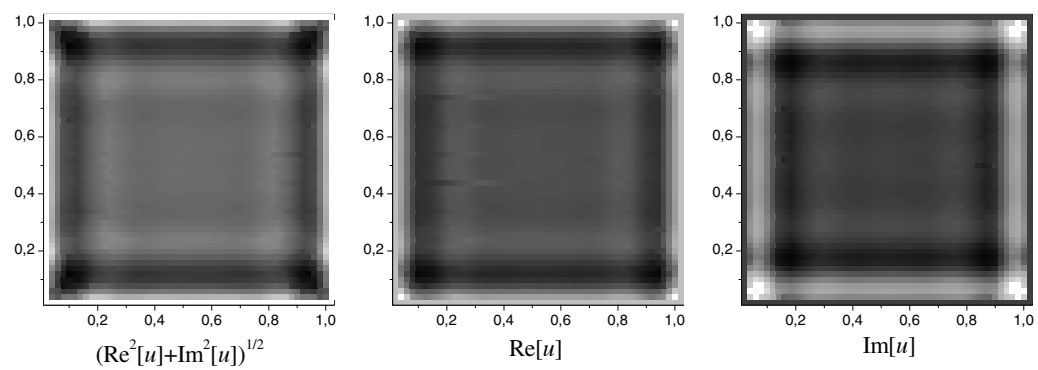

Fig. 11. Distributions of absolute value, real part and imaginary part of $u(y, z)$ at $v=5 \times 10^{7} \mathrm{~Hz}$.

The model approach applied in this paper provides certain advantages over the phenomenological approaches. In particular, the Mitsui model allowed us to get $c_{44}^{E}(\omega)$ elastic constant, for which no experimental data are available, but which is needed for the study of piezoelectric resonance. The Mitsui model describes physical properties of other ferroelectric compounds, including $\mathrm{RbHSO}_{4}, \mathrm{NH}_{4} \mathrm{HSO}_{4}$ (which are piezoelectric in the ferroelectric phase) and others. The results obtained here can be applied to these and other ferroelectric compounds with piezoelectric effect.

\section{References}

Akao, H. \& Sasaki, T. (1955). Dielectric dispersion of Rochelle salt in the microwave region, J. Chem. Phys. 23(12): 2210-2214.

Authier, A. (ed.) (2003). Physical Properties of Crystals, Vol. D of International Tables for Crystallography, Kluwer Academic Publishers, Dordrecht.

Beige, H. \& Kühnel, A. (1984). Electromechanical coefficients at ferroelectric phase transitions, Rochelle salt and $\mathrm{RbHSO}_{4}$, Phys. Status Solidi A 84: 433-437.

Bellaiche, L., García, A. \& Vanderbilt, D. (2000). Finite-temperature properties of $\mathrm{Pb}\left(\mathrm{Zr}_{1-x} \mathrm{Ti}_{x}\right) \mathrm{O}_{3}$ alloys from first principles, Phys. Rev. Lett. 84(23): 5427-5430.

Bronowska, W. J. (1981). Thermal expansion and phase transitions of sodium potassium tartrate tetrahydrate (RS), J. Appl. Crystallogr. 14: 203-207.

Cady, W. (1964). Piezoelectricity: An introduction to the theory and applications of electromechanical phenomena in crystals, Dover, New York.

Deyda, H. (1967). Das temperaturrerhalten der dielectrizitätskonstante von seignettesaltz im mikrowellengebiet, Z. Naturforsch 22a: 1139-1140.

Fu, H. \& Cohen, R. E. (2000). Polarization rotation mechanism for ultrahigh electromechanical response in single-crystal piezoelectrics, Nature 403: 281-284.

Garcia, A. \& Vanderbilt, D. (1998). Electromechanical behavior of $\mathrm{BaTiO}_{3}$ from first principles, Appl. Phys. Lett. 72(23): 2981-2984.

Görbitz, C. H. \& Sagstuen, E. (2008). Potassium sodium $(2 r, 3 r)$-tartrate tetrahydrate: the paraelectric phase of Rochelle salt at 105 K, Acta Cryst. E 64(4): m507-m508.

Guo, R., Cross, L. E., Park, S.-E., Noheda, B., Cox, D. E. \& Shirane, G. (2000). Origin of the high piezoelectric response in $\mathrm{PbZr}_{1-x} \mathrm{Ti}_{x} \mathrm{O}_{3}$, Phys. Rev. Lett. 84(23): 5423-5425.

Hlinka, J., Kulda, J., Kamba, S. \& Petzelt, J. (2001). Resonant soft mode in Rochelle salt by inelastic neutron scattering, Phys. Rev. B 63: 052102-4. 
Jäckle, W. (1960). Dielectric propertiesl of the Rochelle salt, Z. Angew. Math. Phys. 12: 148-150. Jona, F. \& Shirane, G. (1965). Ferroelectric Crystals, Dover Publications, New York.

Kołodziej, H. A. (1975). Dielectric relaxation in ferroelectrics of order-disorder type, Vol. 2, The Chemical Society, Burlington House, London, pp. 249-287.

Levitskii, R. R., Andrusyk, A. \& Zachek, I. R. (2010). Dynamics of the rochelle salt $\mathrm{NaKC}_{4} \mathrm{H}_{4} \mathrm{O}_{6} \cdot 4 \mathrm{H}_{2} \mathrm{O}$ crystal studied within the Mitsui model extended by piezoelectric interaction and transverse field, Condens. Matter Phys. 13(1): 13705.

Levitskii, R. R., Zachek, I. R. \& Andrusyk, A. (2010). The thermodynamics of the Rochelle salt $\mathrm{NaKC}_{4} \mathrm{H}_{4} \mathrm{O}_{6} \cdot 4 \mathrm{H}_{2} \mathrm{O}$ crystal studied within the Mitsui model extended by piezoelectric interaction and transverse field, J. Phys. Stud. 14(3): 3701.

Levitskii, R. R., Zachek, I. R. \& Moina, A. P. (2005). Monoclinic elastic and piezoelectric properties of Rochelle salt. Description within the modified Mitsui model, Condens. Matter Phys. 8(4(44)): 881-890.

Levitskii, R. R., Zachek, I. R., Vdovych, A. S. \& Stasyuk, I. V. (2009). The effect of transverse electric fields on dielectric, piezoelectric, elastic and thermal properties of the Rochelle salt $\mathrm{Rb}\left(\mathrm{H}_{x} \mathrm{D}_{1-x}\right)_{2} \mathrm{PO}_{4}$, Condens. Matter Phys. 12(2): 295-317.

Levitskii, R. R., Zachek, I. R., Verkholyak, T. M. \& Moina, A. P. (2003). Dielectric, piezoelectric, and elastic properties of the Rochelle salt $\mathrm{NaKC}_{4} \mathrm{H}_{4} \mathrm{O}_{6} \cdot 4 \mathrm{H}_{2} \mathrm{O}$ : A theory, Phys. Rev. $B$ 67(17): 174112.

Mason, W. P. (1939). A dynamic measurement of the elastic, electric and piezoelectric constants of rochelle salt, Phys. Rev. 55(8): 775-789.

Mason, W. P. (1950). Piezoelectric Crystals and Their Application to Ultrasonics, Van Nostrand, New York.

Yu. Serdobolskaya, O. (1996). Elastic properties of the Rochelle salt, the system with double critical point, Sol. Stat. Phys. 38(5): 1529-1535.

Mitsui, T. (1958). Theory of the ferroelectric effect in Rochelle salt, Phys. Rev. 111(5): 1529-1567.

Moina, A. P., Levitskii, R. R. \& Zachek, I. R. (2005). Piezoelectric resonance and sound attenuation in the Rochelle salt $\mathrm{NaKC}_{4} \mathrm{H}_{4} \mathrm{O}_{6} \cdot 4 \mathrm{H}_{2} \mathrm{O}$, Phys. Rev. B 71(13): 134108.

Mueller, H. (1935). Properties of Rochelle salt, Phys. Rev. 47(2): 175-191.

Müser, H. E. \& Potthaest, J. (1967). Zum dielektrischen verhalten von seignettesalz im bereich der decimeter- und zentimeterwellen, Phys. Status Solidi 24(1): 109-113.

Noda, N., Nozaki, R. \& Shiozaki, Y. (2000). Calorimetric measurements of the phase transition in Rochelle salt-ammonium Rochelle salt mixed crystals, Phys Rev B 62: 12040-12044.

Park, S. E. \& Shrout, T. R. (1997). Ultrahigh strain and piezoelectric behavior in relaxor based ferroelectric single crystals, J. Appl. Phys. 82(4): 1804-1811.

Pereverzeva, L. P. (1974). Dynamic properties and dispersion of the dielectric permittivity in the ferroelectrics with hydrogen bonds, Kaunas, pp. 223-227.

Poplavko, Y. M., Meriakri, V. V., Pereverzeva, L. P., Alesheckin, V. V. \& Molchanov, V. I. (1974). Investigation of the dielectric properties of Rochelle salt at freguency 1-300 GHz, Sov. Phys. Solid State 15(8): 1672-1673.

Saito, Y., Takao, H., Tani, T., Nonoyama, T., Takatori, K., Homma, T., Nagaya, T. \& Nakamura, M. (2004). Lead-free piezoceramics, Nature 432: 84-87.

Sandy, F. \& Jones, R. V. (1968). Dielectric relaxation of Rochelle salt, Phys. Rev. 168(2): 481-493.

Shiozaki, Y., Nakamura, E. \& Mitsui, T. (eds) (2006). 67A-1 $\mathrm{NaKC}_{4} \mathrm{H}_{4} \mathrm{O}_{6} \cdot 4 \mathrm{H}_{2} \mathrm{O}$ [F], Vol. 36C of Landolt-Börnstein - Group III Condensed Matter Numerical Data and Functional Relationships in Science and Technology, Springer-Verlag, Berlin. 
Shiozaki, Y., Shimizu, K. \& Nozaki, R. (2001). Disordered feature in Rochelle salt, Ferroelectrics 261: 239-244.

Shiozaki, Y., Shimizu, K., Suzuki, E. \& Nozaki, R. (1998). Structural change in the paraelectric phase of Rochelle salt, J. Korean Phys. Soc 32: S192-S194.

Shuvalov, L. A. (ed.) (1988). Modern Crystallography IV: Physical Properties of Crystals, Springer-Verlag, Berlin.

Solans, X., Gonzalez-Silgo, C. \& Ruiz-Perez, C. (1997). A structural study on the Rochelle salt, J. Solid State Chem. 131(2): 350-357.

Stasyuk, I. V. \& Velychko, O. V. (2005). Theory of Rochelle salt: Beyond the Mitsui model, Ferroelectrics 316(1): 51-58.

Taylor, W., Lockwood, D. J. \& Labbe, H. J. (1984). Raman spectroscopy and dielectric constants of ferroelectric Rochelle salt and calcium tartrate, J. Phys. C.: Solid State Phys. 17: 3685-3699.

Ubbelohde, A. R. \& Woodward, I. (1946). Crystals structural and thermal characteristics. The role of hydrogen bonds in Rochelle salt, Proc. Roy. Soc. 185: 448-452.

Volkov, A. A., Kozlov, G. V. \& Lebedev, S. P. (1980). Submillimeter dielectric spectra of Rochelle salt, Sov. Phys. JETP 52(4): 722-726.

Žekš, B., Shukla, G. G. \& Blinc, R. (1971). Dynamics of ferroelectric Rochelle salt, Phys. Rev. B 3(7): 2306-2309.

Yurin, A. (1965). Bull. Acad. Sci. USSR, Phys. Ser. (Engl. Transl.) 29: 2001.

Zhang, S., Randall, C. A. \& Shrout, T. R. (2003). High Curie temperature piezocrystals in the $\mathrm{BiScO}_{3}-\mathrm{PbTiO}_{3}$ perovskite system, Appl. Phys. Lett. 83: 3150-3152. 


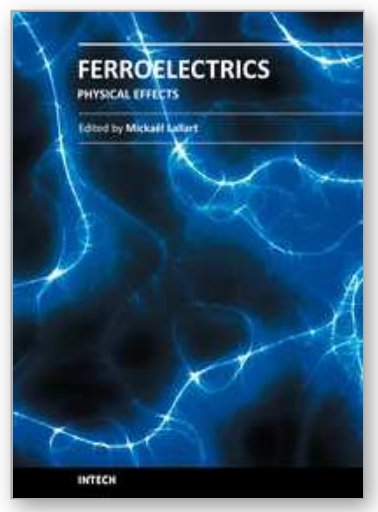

\author{
Ferroelectrics - Physical Effects \\ Edited by Dr. MickaÃ «l Lallart
}

ISBN 978-953-307-453-5

Hard cover, 654 pages

Publisher InTech

Published online 13, June, 2011

Published in print edition June, 2011

Ferroelectric materials have been and still are widely used in many applications, that have moved from sonar towards breakthrough technologies such as memories or optical devices. This book is a part of a four volume collection (covering material aspects, physical effects, characterization and modeling, and applications) and focuses on the underlying mechanisms of ferroelectric materials, including general ferroelectric effect, piezoelectricity, optical properties, and multiferroic and magnetoelectric devices. The aim of this book is to provide an up-to-date review of recent scientific findings and recent advances in the field of ferroelectric systems, allowing a deep understanding of the physical aspect of ferroelectricity.

\title{
How to reference
}

In order to correctly reference this scholarly work, feel free to copy and paste the following:

Andriy Andrusyk (2011). Piezoelectric Effect in Rochelle Salt, Ferroelectrics - Physical Effects, Dr. MickaÃ «l Lallart (Ed.), ISBN: 978-953-307-453-5, InTech, Available from:

http://www.intechopen.com/books/ferroelectrics-physical-effects/piezoelectric-effect-in-rochelle-salt

\section{INTECH}

open science | open minds

\section{InTech Europe}

University Campus STeP Ri

Slavka Krautzeka 83/A

51000 Rijeka, Croatia

Phone: +385 (51) 770447

Fax: +385 (51) 686166

www.intechopen.com

\section{InTech China}

Unit 405, Office Block, Hotel Equatorial Shanghai

No.65, Yan An Road (West), Shanghai, 200040, China

中国上海市延安西路65号上海国际贵都大饭店办公楼405单元

Phone: +86-21-62489820

Fax: +86-21-62489821 
(C) 2011 The Author(s). Licensee IntechOpen. This chapter is distributed under the terms of the Creative Commons Attribution-NonCommercialShareAlike-3.0 License, which permits use, distribution and reproduction for non-commercial purposes, provided the original is properly cited and derivative works building on this content are distributed under the same license. 